\title{
Mechanical characterization and inclusion based boundary element modeling of lightweight concrete containing foam particles
}

\author{
G. Song ${ }^{\mathrm{a}}$, L. Wang ${ }^{\mathrm{a}}$, L. Deng ${ }^{\mathrm{a}}$, H.M. Yin ${ }^{\mathrm{a}, *}$ \\ ${ }^{a}$ Department of Civil Engineering and Engineering Mechanics, Columbia University, 610 Seeley W. Mudd 500 West \\ 120th Street, New York, NY 10027
}

\begin{abstract}
This paper investigates the effective material properties of lightweight concrete containing polymer foam particles through both experiments and numerical modeling. Different volume fractions of foam particles have been added to cement paste to fabricate lightweight concrete samples. To interpret the experimental results, an inclusion method based boundary integral equation has been proposed. The effect of the material mismatch between the foam particles and the cement matrix can be simulated by an eigenstrain, which is a fictitious nonmechanical strain. Due to the interaction between particles and boundary effect, the eigenstrain on a particle is not uniform. An asymptotic analysis shows that a quadratic distribution of the eigenstrain over each particle provides very good accuracy. Since the discretization of particles is not needed due to applying an analytic form of eigenstrain field, a large number of spherical inhomogeneities can be simulated and the local field can be calculated in a lightweight concrete sample. The formulation has been implemented in a software package for the simulation of material samples and has been verified with the finite element method for two top-down particles embedded in a cylinder. The simulation results based on idealized microstructure exhibit very good agreement with the experimental results of the effective elastic moduli. A simple and emprical study also predicts the strengths of the composites very well. The developed algorithm can be used for virtual mechanical experiments of particulate composites.
\end{abstract}

Keywords: Elastic boundary value problem, Boundary element method, Eshelby's equivalent inclusion method, Representative volume element, Lightweight concrete, Micromechanics

\section{Introduction}

Structural lightweight concrete (LWC) has an in-place density on the order of 90 to $115 \mathrm{lb} / \mathrm{ft}^{3}$ which is smaller than normal weight concrete with a density in the range of 140 to $150 \mathrm{lb} / \mathrm{ft}^{3}$. The primary use of structural lightweight concrete is to reduce the dead load of a concrete structure,

${ }^{*}$ Corresponding author. Tel.: +1 212851 1648; Fax: +1 2128546267

Email address: yin@civil. columbia. edu (H.M. Yin)

Preprint submitted to Mechanics of Materials

July 21, 2015

(C) 2015. This manuscript version is made available under the Elsevier user license http://www.elsevier.com/open-access/userlicense/1.0/ 
so that the structural designers can reduce the size of columns, beams and other load bearing elements. While lightweight concrete may cost more per cubic yard than normal weight concrete, the total structure may cost less as a result of reduced size of load bearing elements and lower foundation costs [15]. Lightweight concrete is mainly classified into two groups: lightweight aggregate concrete(LWAC) and autoclaved aerated concrete(AAC). Many types of lightweight aggregate are used in industry manufacture. The early lightweight aggregates (LWAs) were made of natural volcanics, such as pumice, scoria, tuff, etc. Lightweight aggregate can be expensive but it still exhibits a relatively high strength compared with another type of lightweight concrete, autoclaved aerated concrete. Autoclaved aerated concrete (AAC) is commonly manufactured from a mixture of portland cement, fly ash (or other sources of silica), quick lime, gypsum, water, and aluminum powder or paste [16] and its strength can be significantly lower than the LWAC alternative.

Recent researchers in lightweight concrete have used recycled polymer foams, for example, expanded polyethylene [1], expanded polystyrene [2] and polyurethanes [3], as an aggregate to produce lightweight concrete. Generally, commingled plastic waste (CPW) is difficult to recycle due to contamination, variability in composition and incompatibility of the various plastics in the waste stream. However it can be used as concrete aggregate, which does not involve the intimate human contact of consumer products. Using the CPW foam particles for lightweight concrete not only provides one way to reduce the concrete density, but also makes it possible to tailor the thermal resistance, acoustic insulation, and durability of the structural components. This technology requires a method to produce foam from commingled plastic waste, accomplished by the variability of plastic waste stream compositions. There exist two basic types of blowing agents to produce polymeric foams [4]. Chemical blowing agents undergo a chemical reaction to produce a gas that causes foaming. Physical blowing agents do not take part in a chemical reaction, but volatile liquids evaporate and make the foam expand. First generation physical blowing agents are mostly outruled because of their negative environmental impact on the ozone layer.

The present research is motivated by manufacturing a hybrid solar roofing panel as Fig.1 [5, 6, 7]. It includes a substrate made of a lightweight concrete panel to provide structural support to the roof load, above which is a functionally graded material layer with gradient thermal conductivity in the thickness direction, which gradually transitions from a well conductive side attached with PV solar cells laminated by a protective layer to another highly insulative side bonded to the lightweight concrete substrate. The water flowing through the FGM layer cools down photovoltaic (PV) cells and harvests the solar heat. Consequently, the efficiency of PV cell is improved while working under a moderate temperature condition; and the water flow through the FGM layer is useful for preheated water systems. The mechanical properties of the lightweight concrete panel are key parameters in panel design and manufacture.

This paper investigates the effect of the volume fraction of polymer foam particles on the effective material properties of lightweight concrete. For simplicity and consistency of the tests, Elemix $\mathrm{XE}$ concrete additive foam particles have been used in all tests. Because the mechanical loading resistance of foam particles is very low, this lightweight concrete could be treated as an aerated concrete. Compared with traditional AAC manufactured by chemical processes, this lightweight concrete is more environment-friendly and affordable in manufacturing. By mixing the cement 


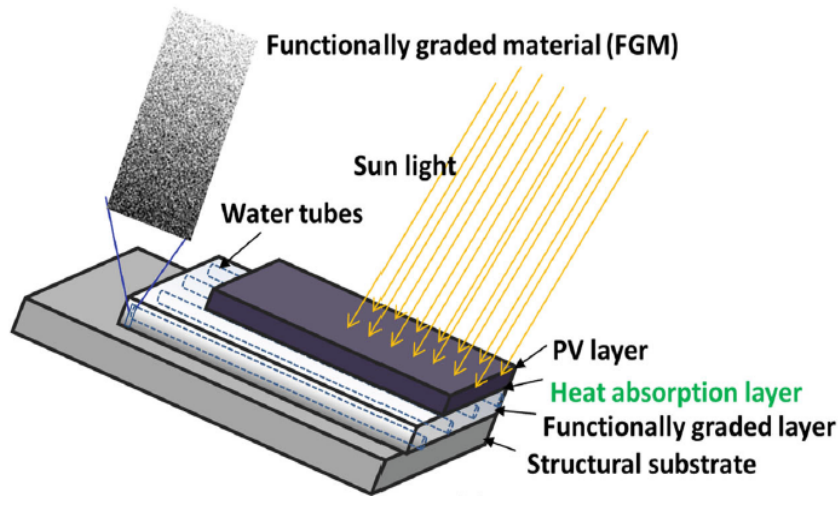

Figure 1: Schematic illustration of the hybrid solar roofing panel prototype

paste with foamed CPW particles, which have a very minimal or negligible cost, a lightweight concrete can be manufactured without any chemical reaction with additives and environmental costs. Moreover, its volume fraction can be easily controlled by weighting the polymer and cement; in contrast, it is very difficult to control the void ratio in AAC. So the variation in quality is less than traditional AAC.

Previous studies on the effective elastic modulus and strength of lightweight concrete are basically about to simply develop empirical formulas by curve fitting the experimental results $[17,18,19]$. Even though much data is available for lightweight aerated concrete, only very few analytical or numerical models exist in the literature to describe the effective mechanical behavior of this porous material due to the difficulty in simulating large number of the particles and the complexity of boundary conditions in a specific finite domain.

Micromechanics-based modeling provides an effective approach to correlating the effective material behavior with the microstructural parameters of composite materials. This lightweight concrete can be treated as a two-phase composite material with the foam particle as dispersed inhomogeneities and cement as the matrix. Given a composite specimen, one can apply a test load on the boundary, which is generally a uniform stress or displacement, to characterize the effective mechanical properties. At the macroscale, the composite can be considered as a homogeneous material; whereas at the microscale, the microstructure is heterogeneous. A representative volume element (RVE) [20, 21, 22, 23] can be used to correlate the effective material behavior at the macroscale to the local mechanical field at the microscale. An RVE for a material point of a continuous mass is a material volume which is statistically representative of the mircostructure in the neighborhood of the infinitesimal material point. The microstructure can be periodic $[24,25$, 26], random, or even a functionally graded materials [27, 28, 29]. Most classical approaches are to investigate the mechanical response of this RVE when far field stress or strain is applied [30,31]. In other words, it assumes that the composite material size is much larger than the particles [22, 23].

Many micromechanical formula have been proposed to predict the effective elastic modulus of composites with different volume fractions. For the Voigt model [32], it assumes the strain on all 
phases is the same in a infinite domain, whereas the Ruess model [33] assumes the stress on all phases is the same. They commonly provide two bounds for the effective mechanical behavior of a composite. Since Eshelby's pioneer work [30, 31] different mciromechanics-based models have been developed, such as the the Mori-Tanaka model [35], the self-consistent model [34, 36], and the generalized self-consistent model [8]. However, the microstructure or particle interactions have not been directly considered in the above models, so that they may provide inaccurate predictions when the particle interaction becomes significant at higher volume fractions.

Numerical methods have been commonly used in the stress analysis of an RVE or a unit cell containing a finite number of particles, which can represent the microstructure of the composite $[11,12]$. However, due to the high computational cost, the material system is limited to a certain scale. For example, Guesv [9] used Monte Carlo realizations to generate the random microstructure of a unit cell including 8,27, and 64 spherical particles and studied the effective moduli of the RVE under the periodic boundary condition. Kanita et al. [10] adopted the Voronoi mosaic model to generate random microstructures for finite element simulations and computed the effective elastic moduli and thermal conductivity considering three types of boundary conditions for different sizes of RVE. They found that a large number of realizations are needed when the size of an RVE is small and stress and displacement distribution is significantly changed for different boundary conditions.

This paper presents a novel method of combining the boundary element method (BEM) and the equivalent inclusion method (EIM) to consider an RVE with a large number of inhomogeneities, which has been explored by Ma and his colleagues [13,14] for two-dimensional inhomogeneity problems. This paper generalizes this method through the software package iBEM to the three dimensional cases. The algorithm is established in this paper using higher order terms of eigenstrain. It provides very accurate results for the local elastic field of a finite domain containing a large number of particles. Since no mesh is needed on the particles, the computational cost can be significantly reduced. The proposed approach can be used to in virtual mechanical experiments for material design and analysis.

In what follows, Section 2 will present the experiments. In Section 3, basic formulation for the inclusion-based BEM(iBEM) will be elaborated. In addition, the detailed computational implementation is provided to establish the global linear equations system. In Section 4, a numerical model is built to simulate a large number of spherical voids distributed in the cement matrix. Very good comparison of the modeling results with the experimental results has been demonstrated. Some conclusions are made in Section 5.

\section{Experiment}

The present lightweight concrete has been fabricated with polymer foam particles and cement with a water-cement ratio at 0.4. The foamed polymer particles, shown in Fig.2 are Elemix XE Concrete Additive. It is a lightweight synthetic particle and each particle is a closed cell sphere of expanded polystyrene (EPS) with a size of $6.4 \mathrm{~mm}$, and density $0.042 \mathrm{~g} / \mathrm{cm}^{3}$. The cement is from LAFARGE, Portland, Type III high-early strength cement. 


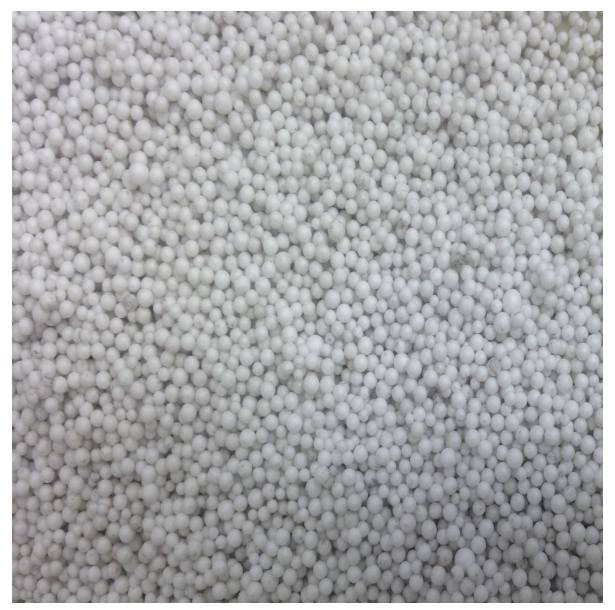

Figure 2: Polymer foam particles with the density of $0.042 \mathrm{~g} / \mathrm{cm}^{3}$ and size of $6.4 \mathrm{~mm}$

Eleven groups of samples have been fabricated for the testing, each group has three replicas. One is used to test the strength; the other two are used to test the Young's modulus and Poisson's ratio. The constituents of material groups are shown in Table 1.

Table 1: Weight of the components for the 11 material groups with different volume fractions

\begin{tabular}{cccc}
\hline Volume fraction $(\%)$ & Water $(\mathrm{g})$ & Cement $(\mathrm{g})$ & Foam particle $(\mathrm{g})$ \\
\hline 0 & 955.01 & 2387.53 & 0.00 \\
5 & 907.26 & 2268.15 & 3.46 \\
10 & 859.51 & 2148.78 & 6.92 \\
15 & 811.76 & 2029.40 & 10.37 \\
20 & 764.01 & 1910.02 & 13.83 \\
25 & 716.6 & 1790.65 & 17.29 \\
30 & 668.51 & 1671.27 & 20.75 \\
35 & 620.76 & 1551.89 & 24.20 \\
40 & 573.01 & 1432.52 & 27.66 \\
45 & 525.26 & 1313.14 & 31.12 \\
50 & 477.51 & 1193.76 & 34.58
\end{tabular}

To fabricate the lightweight concrete containing polymer foam particles, cement is firstly mixed with water in a mixer for 3 minutes, and then polymer is slowly poured in and stirred at a low speed for 6 minutes to achieve good workability as shown in Fig.3(a). At the end of the mixing process, the material attached to the stirring rod will be carefully cleaned and replaced in the mixer in order to maintain the polymer and cement ratio in the final material samples. Notice that the fresh lightweight concrete cannot be processed with the vibration table, because the vibration would cause the separation of the polymer and concrete. A steel bar can be used to stir the sample to 
facilitate compaction and reduce the amount of air bubbles. The specimens are demolded at 24 hours and then cured in water tank at the temperature of $22{ }^{\circ} \mathrm{C}$ for 7 days as in Fig.3(b). Then they are placed in the laboratory environment until tested. Before testing, the sample is fully dried out to meet the ASTM international C495-07 and C39/C39M-09 [37]. Then each lightweight concrete cylinder is capped to ensure that the test specimen has a smooth, parallel, uniform bearing surface that is perpendicular to the applied axial load during the compressive strength test.

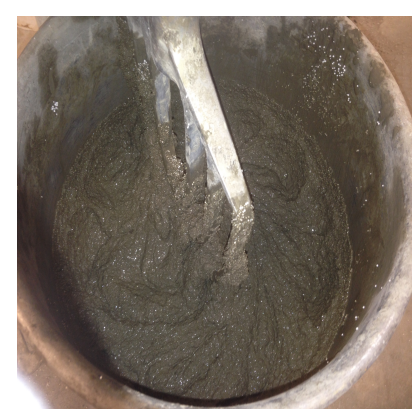

(a)

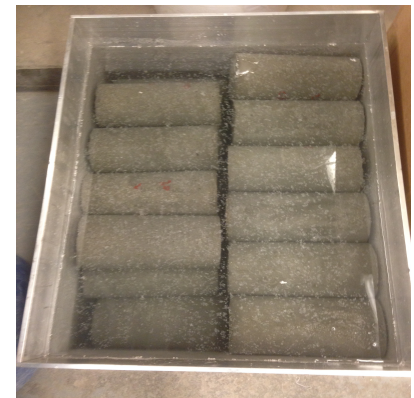

(b)

Figure 3: Specimen preparation - (a) cement mixing with polymers in mixer and (b) lightweight concrete curing in the water

Compressive strength tests have been performed by a Instron 1500HDX Universal Testing Machine in accordance with ASTM C495-07 and C39/C39M-09. A universal testing machine has been used as shown in Fig.4(b). The testing load has been controlled by force with the loading rate at $0.225 \mathrm{lbs} / \mathrm{sec}$. The vertical and horizontal strains of the sample versus the compressive load have also been measured with the strain gauge encircling the sample as shown in Fig.4(a).

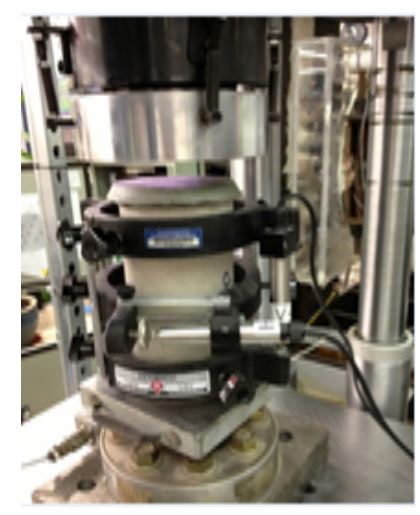

(a)

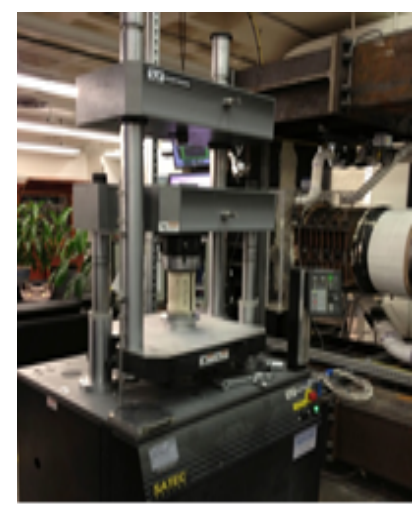

(b)

Figure 4: Experimental test configuration for testing - (a) Young's Modulus and Poisson's ratio and (b) compressive strength

Through the data analysis, the density, averaged Young's modulus, standard deviation of the 
Young's modulus and Poisson's ratio of the test specimens are obtained with respect to different volume fractions as Table 2. Notice that the data of density and Young's modulus for the sample with volume fraction $\phi=0.1$ is out of the trend, which implies some inconsistencies/misoperations may have occurred in the material preparation. Therefore, we will treat it as an outlier and it will not be counted in later analysis.

Table 2: Test results for lightweight concrete specimens with different volume fractions

\begin{tabular}{cccccc}
\hline volume fraction & $\begin{array}{c}\text { density } \\
\left(\mathrm{Kg} / \mathrm{m}^{3}\right)\end{array}$ & $\begin{array}{c}\text { strength } \\
(\mathrm{psi})\end{array}$ & Poisson's ratio & $\begin{array}{c}\text { averaged Young's } \\
\text { modulus }(\mathrm{GPa})\end{array}$ & $\begin{array}{c}\text { standard deviation } \\
(\mathrm{GPa})\end{array}$ \\
\hline 0.0 & 2008.0 & 5481.5 & 0.25 & 16.9 & 0.44 \\
0.05 & 1854.2 & 5669.3 & 0.28 & 13.7 & 0.05 \\
0.1 & 1686.9 & 2950.5 & 0.30 & 10.0 & 0.20 \\
0.15 & 1625.6 & 3783.6 & 0.35 & 12.1 & 0.11 \\
0.20 & 1558.2 & 3027.6 & 0.28 & 8.6 & 0.07 \\
0.25 & 1435.2 & 2421.7 & 0.31 & 8.7 & 0.62 \\
0.30 & 1328.3 & 2457.4 & 0.31 & 6.9 & 0.35 \\
0.35 & 1211.0 & 1866.6 & 0.29 & 6.0 & 0.52 \\
0.40 & 1107.2 & 1517.1 & 0.33 & 4.9 & 0.06 \\
0.45 & 1011.3 & 1249.6 & 0.24 & 4.1 & 0.09 \\
0.50 & 945.5 & 1038.5 & 0.26 & 3.9 & 0.10
\end{tabular}

\section{Inclusion based boundary integral formulation}

To interpret the test results, in this section, an equivalent inclusion method (EIM) based boundary integral formulation will be presented to investigate multiple inhomogeneities embedded in a matrix with finite domain under the test loading. Then, a global equation system can be assembled to solve the unknown boundary displacements and the eigenstrain in each inhomogeneity. With that informations, the local displacement and stress fields can be derived by the Green's function technique.

\subsection{Basic formulation of boundary element method (BEM)}

Consider a domain $D$ is subjected to body force $\boldsymbol{b}$ and surface traction $\boldsymbol{t}$. In elasticity, the virtual work principle reads

$$
\int_{\Omega} \sigma_{i j} u_{i, j}^{\prime} \mathrm{d} \mathbf{x}=\int_{\partial \Omega} t_{i} u_{i}^{\prime} \mathrm{d} \mathbf{x}+\int_{\Omega} b_{i} u_{i}^{\prime} \mathrm{d} \mathbf{x}
$$

where $u_{i}^{\prime}$ is an admissible virtual displacement.

Use the true displacement $u_{i}$ as a virtual displacement. Let $\sigma_{i j}^{\prime}$ be a new stress state associated with another equilibrium state caused by $\boldsymbol{t}^{\prime}$ and $\boldsymbol{b}^{\prime}$. Applying the virtual work principle in the above equation, one can obtain 


$$
\int_{\partial D} t_{i} u_{i}^{\prime} \mathrm{d} \mathbf{x}+\int_{D} b_{i} u_{i}^{\prime} \mathrm{d} \mathbf{x}=\int_{\partial D} t_{i}^{\prime} u_{i} \mathrm{~d} \mathbf{x}+\int_{D} b_{i}^{\prime} u_{i} \mathrm{~d} \mathbf{x}
$$

which is the well-known Maxwell-Betti's reciprocal theorem. The two loading cases are shown in Fig.5.

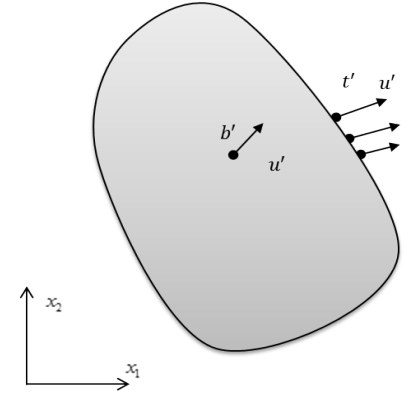

(a)

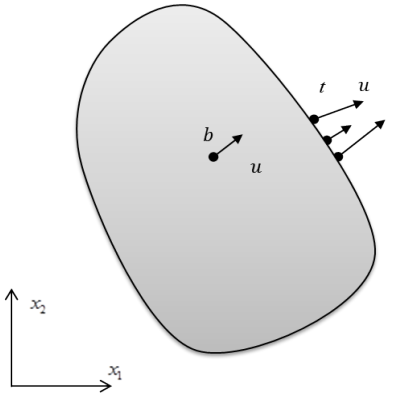

(b)

Figure 5: Application of the Betti reciprocal theorem for two loading cases

Using the equilibrium $b_{i}^{\prime}=-\sigma_{j i, j}^{\prime}$, one can obtain the following equation:

$$
\int_{D} \sigma_{j i, j}^{\prime} u_{i} \mathrm{~d} \mathbf{x}+\int_{D} b_{i} u_{i}^{\prime} \mathrm{d} \mathbf{x}=\int_{\partial D} t_{i}^{\prime} u_{i} \mathrm{~d} \mathbf{x}-\int_{\partial D} t_{i} u_{i}^{\prime} \mathrm{d} \mathbf{x}
$$

Use the fundamental solution to the following equilibrium equation for the new stress state under a unit point force at $\mathbf{x}^{\prime}$,

$$
\sigma_{j i, j}^{\prime}(\mathbf{x})+\delta_{i}\left(\mathbf{x}-\mathbf{x}^{\prime}\right)=0
$$

where $\delta_{i}\left(\mathbf{x}-\mathbf{x}^{\prime}\right)$ is the Dirac delta function indicating a unit point force in the $i$ direction acting at position $\mathbf{x}^{\prime}$ with the component $d_{i}$. Therefore, $d_{i}$ exhibits the following properties.

$$
\left\{\begin{array}{l}
\int_{D} \delta_{i}\left(\mathbf{x}-\mathbf{x}^{\prime}\right) \mathrm{d} \mathbf{x}=d_{i} \\
|\mathbf{d}|=\sqrt{d_{i} d_{i}}=1
\end{array}\right.
$$

This fictitious stress state can be obtained by the classic solution for an infinite domain containing a point force associated with the corresponding stress vector on the boundary. Using the property of the Dirac delta function, one can write

$$
\int_{D} \sigma_{j i, j}^{\prime}(\mathbf{x}) u_{i}(\mathbf{x}) \mathrm{d} \mathbf{x}=-\int_{D} \delta_{i}\left(\mathbf{x}-\mathbf{x}^{\prime}\right) u_{i}(\mathbf{x}) \mathrm{d} \mathbf{x}=-d_{i} u_{i}\left(\mathbf{x}^{\prime}\right)
$$

Now, to solve the displacement at any point $\mathbf{x}^{\prime}$, one can use the fundamental solution for a fictitious unit point force at that point. The fictitious elastic field will depend on both variables of the fictitious loading point $\mathbf{x}^{\prime}$ and the field point $\mathbf{x}$.

Combining Eqs.(3) and (5), one can obtain the displacement at any point $\mathbf{x}^{\prime}$ as follows 


$$
u_{i}\left(\mathbf{x}^{\prime}\right) d_{i}=\int_{\partial D} u_{i}^{\prime}\left(\mathbf{x}, \mathbf{x}^{\prime}\right) t_{i}(\mathbf{x}) \mathrm{d} \mathbf{x}-\int_{\partial D} t_{i}^{\prime}\left(\mathbf{x}, \mathbf{x}^{\prime}\right) u_{i}(\mathbf{x}) \mathrm{d} \mathbf{x}+\int_{D} u_{i}^{\prime}\left(\mathbf{x}, \mathbf{x}^{\prime}\right) b_{i}(\mathbf{x}) \mathrm{d} \mathbf{x}
$$

Using the Green's function technique, the fictitious elastic field can be obtained as follows

$$
\begin{aligned}
& u_{j}^{\prime}\left(\mathbf{x}, \mathbf{x}^{\prime}\right)=G_{i j}\left(\mathbf{x}, \mathbf{x}^{\prime}\right) d_{i} \\
& t_{j}^{\prime}\left(\mathbf{x}, \mathbf{x}^{\prime}\right)=t_{i j}\left(\mathbf{x}, \mathbf{x}^{\prime}\right) d_{i}
\end{aligned}
$$

where $G_{i j}\left(\mathbf{x}, \mathbf{x}^{\prime}\right)$ and $t_{i j}\left(\mathbf{x}, \mathbf{x}^{\prime}\right)$ represent the displacement and traction, respectively, in the $j$ direction at $\mathbf{x}$ caused by a unit point force $f_{i}$ acting at $\mathbf{x}^{\prime}$ in the $i$ direction.

$$
\begin{gathered}
G_{i j}\left(\mathbf{x}, \mathbf{x}^{\prime}\right)=\frac{1}{16 \pi(1-v) \mu}\left[\frac{(3-4 v)}{r} \delta_{i j}+\frac{r_{, i} r_{, j}}{r}\right] \\
t_{i j}\left(\mathbf{x}, \mathbf{x}^{\prime}\right)=\frac{-1}{8 \pi(1-v) r^{2}}\left[\left((1-2 v) \delta_{i j}+3 r_{, i} r_{, j}\right) r_{, m} n_{m}-(1-2 v)\left(r_{, i} n_{j}-r_{, j} n_{i}\right)\right]
\end{gathered}
$$

where $r=\left|\mathbf{x}-\mathbf{x}^{\prime}\right|, r_{, i}=\frac{\partial\left(x_{m} x_{m}\right)^{\frac{1}{2}}}{\partial x_{j}}, r_{, i j}=\frac{\partial^{2}\left(x_{m} x_{m}\right)^{\frac{1}{2}}}{\partial x_{i} \partial x_{j}}$ and $\boldsymbol{n}$ is the unit normal vector. Notice that one can easily see the reciprocal property of the above tensors, i.e.

$$
G_{i j}\left(\mathbf{x}, \mathbf{x}^{\prime}\right)=G_{i j}\left(\mathbf{x}^{\prime}, \mathbf{x}\right) \text { and } t_{i j}\left(\mathbf{x}, \mathbf{x}^{\prime}\right)=t_{i j}\left(\mathbf{x}^{\prime}, \mathbf{x}\right)
$$

Finally, the following boundary integral equation can be obtained:

$$
d_{i} u_{i}\left(\mathbf{x}^{\prime}\right)=d_{i} \int_{\partial D} G_{i j}\left(\mathbf{x}, \mathbf{x}^{\prime}\right) t_{j}(\mathbf{x}) \mathrm{d} \mathbf{x}-d_{i} \int_{\partial D} t_{i j}\left(\mathbf{x}, \mathbf{x}^{\prime}\right) u_{j}(\mathbf{x}) \mathrm{d} \mathbf{x}+d_{i} \int_{D} G_{i j}\left(\mathbf{x}, \mathbf{x}^{\prime}\right) b_{j}(\mathbf{x}) \mathrm{d} \mathbf{x}
$$

Therefore, to obtain the displacement at any point $\mathbf{x}^{\prime}$ in a specific direction, one can apply a unit point force along that direction represented by $d_{i}$ and derive the displacement through the above integrals of $\mathbf{x}$. For the simplicity of notations, we can switch $\mathbf{x}$ and $\mathbf{x}^{\prime}$ in the above equation, so that $\mathbf{x}$ ' stands for the integral variable and $\mathbf{x}$ the point to solve for displacement. In addition, the above equation is applicable to an arbitrary fictitious unit force, so that $d_{i}$ at the both sides of the above equation can be eliminated. Therefore, one can obtain

$$
u_{i}(\mathbf{x})=\int_{\partial D} G_{i j}\left(\mathbf{x}, \mathbf{x}^{\prime}\right) t_{j}\left(\mathbf{x}^{\prime}\right) \mathrm{d} \mathbf{x}^{\prime}-\int_{\partial D} t_{i j}\left(\mathbf{x}, \mathbf{x}^{\prime}\right) u_{j}\left(\mathbf{x}^{\prime}\right) \mathrm{d} \mathbf{x}^{\prime}+\int_{D} G_{i j}\left(\mathbf{x}, \mathbf{x}^{\prime}\right) b_{j}\left(\mathbf{x}^{\prime}\right) \mathrm{d} \mathbf{x}^{\prime}
$$

where the reciprocal property in Eq.(11) is used. For an equilibrium state in the absence of body force, the last term does not exist, so that the displacement can be obtained by the two boundary integral terms. In this case the expression of the stress tensor can be obtained: 


$$
\sigma_{i j}=-\int_{\partial D} R_{i j m} t_{m} \mathrm{~d} \mathbf{x}+\int_{\partial D} S_{i j m} u_{m} \mathrm{~d} \mathbf{x}
$$

where

$$
\begin{gathered}
R_{i j m}=\frac{-1}{8 \pi(1-v)}\left[\frac{3 r_{, i} r_{, j} r_{m}}{r^{2}}+\frac{(1-2 v)\left(\delta_{m i} r_{, j}+\delta_{m j} r_{, i}-\delta_{i j} r_{, m}\right)}{r^{2}}\right] \\
S_{i j m}=\frac{-3 \mu r_{, p} n_{p}}{4 \pi(1-v) r^{3}}\left[(1-2 v) r_{, m} \delta_{i j}+v\left(r_{, i} \delta_{m j}+r_{, j} \delta_{m i}\right)-5 r_{, m} r_{, i} r_{, j}\right] \\
\frac{-\mu}{4 \pi(1-v) r^{3}}\left[3 v\left(r_{, j} r_{m} n_{i}+r_{, i} r_{, m} n_{j}\right)+(1-2 v)\left(3 r_{, i} r_{, j} n_{m}+\delta_{m i} n_{j}+\delta_{m j} n_{i}\right)-(1-4 v) \delta_{i j} n_{m}\right]
\end{gathered}
$$

\subsection{The inclusion problem for an unbound domain}

Consider an unbound homogeneous elastic domain $D$ where an inclusion is embedded in a subdomain $\Omega$, where an eigenstrain rate $\varepsilon_{i j}^{*}(\mathbf{x})$ is applied on $\Omega$ but is zero on $D-\Omega$. The elastic modulus in the inclusion and the rest (matrix) is the same. The term of "inclusion" is to differentiate another counterpart of "inhomogeneity". An inhomogeneity is a subdomain in a homogeneous elastic body with different material properties from the rest.

Consider an ellipsoidal inclusion $\Omega$ embedded in a homogeneous elastic domain, where the ellipsoidal inclusion is defined by the function $\frac{x_{1}^{2}}{a_{1}^{2}}+\frac{x_{2}^{2}}{a_{2}^{2}}+\frac{x_{3}^{2}}{a_{3}^{2}} \leq 1$ with $a_{i}(i=1,2,3)$ being the three principal half axes of the ellipsoid. The elastic modulus is the same in the inclusion and the matrix, written as $C$. An eigenstrain $\varepsilon_{k l}^{*}(\mathbf{x})$ is distributed in the domain $\Omega$, but are zero in the matrix.

Based on the homogeneous constitutive law, the stress can be expressed as

$$
\sigma_{i j}(\mathbf{x})=C_{i j k l}\left[\varepsilon_{k l}(\mathbf{x})-\varepsilon_{k l}^{*}(\mathbf{x})\right] \quad \mathbf{x} \in \Omega
$$

Using Eq.(17), one can write the equilibrium equation in the absence of body force as:

$$
C_{i j k l} \varepsilon_{k l, i}(\mathbf{x})=C_{i j k l} \varepsilon_{k l, i}^{*}(\mathbf{x}) \quad \mathbf{x} \in \Omega
$$

Notice that the right hand side of the equation can be mathematically treated as a body force, i.e.

$$
b_{j}(\mathbf{x})=-C_{i j k l} \varepsilon_{k l, i}^{*}(\mathbf{x}) .
$$

Using the Green's function technique or the fundamental solution [42, 39], one can write the displacement fields in the domain $D$ in terms of the integrals of the eigenstrain $\varepsilon_{k l}^{*}$ with the tensorial Green's function $\mathbf{G}$ as follows:

$$
u_{m}(\mathbf{x})=-\int_{\Omega} C_{i j k l} \frac{\partial \varepsilon_{k l}^{*}}{\partial x_{i}^{\prime}}\left(\mathbf{x}^{\prime}\right) G_{j m}\left(\mathbf{x}, \mathbf{x}^{\prime}\right) \mathrm{d} \mathbf{x}^{\prime}
$$


Using the Gauss' theorem, Eq.(19) can be rewritten as

$$
u_{m}(\mathbf{x})=-\int_{\Omega} C_{i j k l} \varepsilon_{k l}^{*}\left(\mathbf{x}^{\prime}\right) G_{j m, i}\left(\mathbf{x}, \mathbf{x}^{\prime}\right) \mathrm{d} \mathbf{x}^{\prime}
$$

where the eigenstrain in the matrix $\varepsilon_{k l}^{*}=0$ is used, and $G_{j m, i}$ is also called the modified Green's function [38].

In the ellipsoidal domain, the eigenstrain are continuously distributed, so that one can expand them in the polynomial form using the Taylor's expansion. Then the integral in Eq.(20) can be explicitly written with the aid of the identities of the integral of $\phi$ and $\psi$ using the elliptic integrals $[22,42,41,39]$. The $\phi$ and $\psi$ are defined as follows

$$
\phi\left(\mathbf{x}, \mathbf{x}^{\prime}\right)=\frac{1}{\left|\mathbf{x}-\mathbf{x}^{\prime}\right|}, \quad \psi\left(\mathbf{x}, \mathbf{x}^{\prime}\right)=\left|\mathbf{x}-\mathbf{x}^{\prime}\right|
$$

In Eq.(9)

$$
G_{i j}\left(\mathbf{x}, \mathbf{x}^{\prime}\right)=\frac{1}{16 \pi(1-v) \mu}\left[\frac{(3-4 v)}{r} \delta_{i j}+\frac{r_{, i} r_{, j}}{r}\right]
$$

In order to integrate the Green's function more conveniently, we rewrite it in terms of $\phi$ and $\psi$ as follows

$$
G_{j m}\left(\mathbf{x}, \mathbf{x}^{\prime}\right)=\frac{1}{4 \pi \mu}\left(\delta_{j m} \phi-\frac{1}{4(1-v)} \frac{\partial^{2} \psi}{\partial x_{j} \partial x_{m}}\right)
$$

Consider the eigenstrain is expanded in a polynomial form as

$$
\varepsilon_{k l}^{*}=\varepsilon_{k l}^{0}+\varepsilon_{k l p}^{1} x_{p}+\ldots . . \quad \mathbf{x} \in \Omega
$$

One can obtain the displacement caused by the eigenstrain as

$$
u_{m}(\mathbf{x})=C_{i j k l} D_{j m i}(\mathbf{x}) \varepsilon_{k l}^{0}+C_{i j k l} D_{j m i p}(\mathbf{x}) \varepsilon_{k l p}^{1}+\ldots \ldots
$$

where

$$
\begin{gathered}
D_{j m i}=\int_{\Omega}-\frac{1}{2}\left(G_{m j, i}+G_{m i, j}\right) \mathrm{d} \mathbf{x}^{\prime}=-\frac{1}{2}\left[\frac{\delta_{m j}}{4 \pi \mu} \Phi_{, i}+\frac{\delta_{m i}}{4 \pi \mu} \Phi_{, j}-\frac{1}{8 \pi \mu(1-v)} \Psi_{, j m i}\right] \\
D_{j m i p}=\int_{\Omega}-\frac{1}{2}\left(G_{m j, i}+G_{m i, j}\right) x_{p}^{\prime} \mathrm{d} \mathbf{x}^{\prime}=-\frac{1}{2}\left[\frac{\delta_{m j}}{4 \pi \mu} \Phi_{p, i}+\frac{\delta_{m i}}{4 \pi \mu} \Phi_{p, j}-\frac{1}{8 \pi \mu(1-v)} \Psi_{p, j m i}\right]
\end{gathered}
$$

in which $\Phi$ and $\Psi$ denote the integrals of $\phi$ and $\psi$ over the ellipsoidal inclusion; and $\Phi_{p}$ and $\Psi_{p}$ denote the integrals of $\phi x_{p}^{\prime}$ and $\psi x_{p}^{\prime}$. These integrals and their derivative terms are explicitly provided in Appendix A of [39]. 


\subsection{The inclusion problem for a bounded domain}

Consider a bounded domain $D$ containing an inclusion $\Omega$ with a continuously distributed eigenstrain, which is subjected to a certain boundary conditions. For simplicity, up to linear terms in the polynomial form are considered for the eigenstrain. The displacement caused by loads on the boundary and eigenstrain in the inclusions can be obtained by the combination of Eqs. (13) and (20) as follows

$$
u_{m}(\mathbf{x})=\int_{\partial D} G_{m j}\left(\mathbf{x}, \mathbf{x}^{\prime}\right) t_{j}\left(\mathbf{x}^{\prime}\right) \mathrm{d} \mathbf{x}^{\prime}-\int_{\partial D} t_{m j}\left(\mathbf{x}, \mathbf{x}^{\prime}\right) u_{j}\left(\mathbf{x}^{\prime}\right) \mathrm{d} \mathbf{x}^{\prime}+C_{i j k l} D_{j m i}(\mathbf{x}) \varepsilon_{k l}^{0}+C_{i j k l} D_{j m i p}(\mathbf{x}) \varepsilon_{k l p}^{1}
$$

The strain can also be obtained by applying the relationship between displacement and strain

$\varepsilon_{m n}(\mathbf{x})=-C_{m n k l}^{-1} \int_{\partial D} R_{k l p}\left(\mathbf{x}, \mathbf{x}^{\prime}\right) t_{p}\left(\mathbf{x}^{\prime}\right) \mathrm{d} \mathbf{x}^{\prime}+C_{m n k l}^{-1} \int_{\partial D} S_{k l p}\left(\mathbf{x}, \mathbf{x}^{\prime}\right) u_{p}\left(\mathbf{x}^{\prime}\right) \mathrm{d} \mathbf{x}^{\prime}+C_{i j k l} D_{j m i n}(\mathbf{x}) \varepsilon_{k l}^{0}+C_{i j k l} D_{j m i n p}(\mathbf{x}) \varepsilon_{k l p}^{1}$

where

$$
\begin{gathered}
D_{j m i n}=-\frac{1}{4}\left[\frac{\delta_{m j}}{4 \pi \mu} \Phi_{, i n}+\frac{\delta_{m i}}{4 \pi \mu} \Phi_{, j n}+\frac{\delta_{n j}}{4 \pi \mu} \Phi_{, i m}+\frac{\delta_{n i}}{4 \pi \mu} \Phi_{, j m}-\frac{1}{4 \pi \mu(1-v)} \Psi_{, i j m n}\right] \\
D_{\text {jmin }}=-\frac{1}{4}\left[\frac{\delta_{m j}}{4 \pi \mu} \Phi_{p, i n}+\frac{\delta_{m i}}{4 \pi \mu} \Phi_{p, j n}+\frac{\delta_{n j}}{4 \pi \mu} \Phi_{p, i m}+\frac{\delta_{n i}}{4 \pi \mu} \Phi_{p, j m}-\frac{1}{4 \pi \mu(1-v)} \Psi_{p, i j m n}\right]
\end{gathered}
$$

and the tensors of $R_{k l p}$ and $S_{k l p}$ have been shown in Eqs. (15) and (16).

For the convenience of derivation later, the first derivative of strain with respect to $\mathbf{x}$ can also be obtained as follows:

$$
\varepsilon_{m n, q}=C_{m n k l}^{-1} \int_{\partial \Omega} R_{k l p q} t_{p}-C_{m n k l}^{-1} \int_{\partial \Omega} S_{k l p q} u_{p}+C_{i j k l} D_{j m i n q} \varepsilon_{k l}^{0}+C_{i j k l} D_{j m i n p q} \varepsilon_{k l p}^{1}
$$

where

$$
\begin{aligned}
R_{k l p q}= & -\frac{1}{8 \pi(1-v)}\left(\frac{3 r_{, k q} r_{, l} r_{, p}}{r^{2}}+\frac{3 r_{, k} r_{, l q} r_{, p}}{r^{2}}+\frac{3 r_{, k} r_{, l} r_{, p q}}{r^{2}}-\frac{6 r_{, k} r_{, l} r_{, p} r_{, q}}{r^{3}}\right) \\
& -\frac{(1-2 v)}{8 \pi(1-v)}\left(\frac{\delta_{p k} r_{, l q}}{r^{2}}+\frac{\delta_{p l} r_{k q}}{r^{2}}-\frac{\delta_{k l} r_{, p q}}{r^{2}}\right)+\frac{(1-2 v)}{4 \pi(1-v)}\left(\frac{\delta_{p k} r_{, l} r_{, q}}{r^{3}}+\frac{\delta_{p l} r_{, k} r_{, q}}{r^{3}}-\frac{\delta_{k l} r_{, p} r_{, q}}{r^{3}}\right)
\end{aligned}
$$




$$
\begin{aligned}
& S_{k l p q}= \frac{9 \mu r_{, n} n_{n} r_{, q}}{4 \pi(1-v) r^{4}}\left[(1-2 v) r_{, p} \delta_{k l}+v\left(r_{, k} \delta_{l p}+r_{, l} \delta_{k p}-5 r_{, p} r_{, k} r_{, l}\right)\right] \\
& \frac{-3 \mu r_{, n q} n_{n}}{4 \pi(1-v) r^{3}}\left[(1-2 v) r_{, p} \delta_{k l}+v\left(r_{, k} \delta_{p l}+r_{, l} \delta_{p k}\right)-5 r_{, p} r_{, k} r_{, l}\right] \\
& \frac{-3 \mu r_{, n} n_{n}}{4 \pi(1-v) r^{3}}\left[(1-2 v) r_{, p q} \delta_{k l}+v\left(r_{, k q} \delta_{p l}+r_{, l q} \delta_{p k}\right)-5\left(r_{, p q} r_{, k} r_{, l}+r_{, p} r_{, k q} r_{, l}+r_{, p} r_{, k} r_{, l q}\right)\right] \\
& \frac{9 \mu v r_{, q}}{4 \pi(1-v) r^{4}}\left(r_{, l} r_{, p} n_{k}+r_{, k} r_{, p} n_{l}\right)-\frac{3 \mu v}{4 \pi(1-v) r^{3}}\left(r_{, l q} r_{, p} n_{k}+r_{, l} r_{, p q} n_{k}+r_{, k q} r_{, p} n_{l}+r_{, k} r_{, p q} n_{l}\right) \\
& \frac{3(1-2 v) \mu r_{, q}}{4 \pi(1-v) r^{4}}\left(3 r_{, k} r_{, l} n_{p}+\delta_{p k} n_{l}+\delta_{p l} n_{k}\right)-\frac{\mu(1-2 v)}{4 \pi(1-v) r^{3}}\left(3 r_{, k q} r_{, l} n_{p}+3 r_{, k} r_{, l q} n_{p}\right)-\frac{3 \mu(1-4 v) r_{, q}}{4 \pi(1-v) r^{4}} \delta_{k l} n_{p} \\
& D_{j m i n q}=\frac{1}{4}\left[\frac{\delta_{m j}}{4 \pi \mu} \Phi_{, i n q}+\frac{\delta_{m i}}{4 \pi \mu} \Phi_{, j n q}+\frac{\delta_{n j}}{4 \pi \mu} \Phi_{, i m q}+\frac{\delta_{n i}}{4 \pi \mu} \Phi_{, j m q}-\frac{1}{4 \pi \mu(1-v)} \Psi_{, i j m n q}\right] \\
& D_{j \text { minpq }}=-\frac{1}{4}\left[\frac{\delta_{m j}}{4 \pi \mu} \Phi_{p, i n q}+\frac{\delta_{m i}}{4 \pi \mu} \Phi_{p, j n q}+\frac{\delta_{n j}}{4 \pi \mu} \Phi_{p, i m q}+\frac{\delta_{n i}}{4 \pi \mu} \Phi_{p, j m q}-\frac{1}{4 \pi \mu(1-v)} \Psi_{p, i j m n q}\right]
\end{aligned}
$$

The explicit form of the above two tensors can also be found in [39].

\subsection{Equivalent inclusion method for a bounded domain}

The equivalent inclusion method (EIM) has been proposed to simulate material mismatch with a prescribed strain, namely eigenstrain, for the material subjected to a load[30, 31]. However, Eshelby's original work focused on the unbound domain with a uniform far field stress, so that the boundary effect has been disregarded. Now consider the inhomogeneity problem for a bound domain $D$ with stiffness $\mathbf{C}^{0}$ containing an inhomogeneity $\Omega$ with stiffness $\mathbf{C}^{1}$, which is subjected to a certain load on the boundary $\partial D$. Because of the material mismatch, the fundamental solution in Subsection 3.1 cannot be directly applied in the above material domain. Owe to Eshelby's original idea of EIM, the inhomogeneity problem can be simulated by an inclusion problem that the inhomogeneity can be replaced by an inclusion with a certain continuously distributed eigenstrain, which satisfies the stress equivalent condition that will be shown in Eq. (31) later. Therefore, the displacement field can be obtained by Eq. (25) with the effects of loading on the boundary.

To simulate the material mismatch, the stress equivalent condition is applied here. For a given inhomogeneity, a local coordinate is set up with the origin located at the inhomogeneity center. Due to continuity of the elastic field, one can expand the total strain by using the Taylor expansion of the local coordinate with the origin at the center of the inhomogeneity,

$$
\varepsilon_{k l}(\mathbf{x})=\varepsilon_{k l}(\mathbf{0})+\varepsilon_{k l, q}(\mathbf{0}) x_{q}+\ldots, \quad \mathbf{x} \in \Omega
$$


In order to make the stresses in the inclusion problem equivalent to those in the inhomogeneity, the following equation should be satisfied.

$$
C_{i j k l}^{0}\left(\varepsilon_{k l}-\varepsilon_{k l}^{*}\right)=C_{i j k l}^{1} \varepsilon_{k l}
$$

By substituting Eq. (23) and Eq. (30) into Eq. (31), one can compare the coefficient for each rank of the $\mathbf{x}$ terms. For example, the following equations for the zeroth and first order termsof $\mathbf{x}$ can be, respectively, written as.

$$
\begin{gathered}
C_{i j k l}^{0}\left[\varepsilon_{k l}(\mathbf{0})-\varepsilon_{k l}^{0}\right]=C_{i j k l}^{1} \varepsilon_{k l}(\mathbf{0}) \\
C_{i j k l}^{0}\left[\varepsilon_{k l, q}(\mathbf{0})-\varepsilon_{k l q}^{1}\right]=C_{i j k l}^{1} \varepsilon_{k l, q}(\mathbf{0})
\end{gathered}
$$

Although only up to linear terms are considered here for simplicity, the method can be extended to higher order terms for higher accuracy. Because introducing one higher order term will lead to one set of equations in the above stress equivalent condition, the problem is mathematically closed. It can be straightforwardly implemented in the next subsection for numerical simulation, in which quadratic terms have already been considered following the above procedure.

The above equation can be extended to multiple inhomogeneities with different sizes, stiffness, or orientations as follows: a local coordinate is set up in the same fashion as above, the eigenstrain on each inhomogeneity can be expanded in the polynomial form at a certain order, which can be different for different inhomogeneities depending on the accuracy requirement. Comparing the coefficient of the stress field on each inhomogeneity in the polynomial form, one can set up a linear equation system similarly to Eqs. (32) and (33). By solving the linear system, the eigenstrain for each particle can be obtained, and then the strain and displacement fields in the domain $D$ can be solved similarly to Eqs. (25) and (26).

Since the ellipsoid is a versatile shape which can represent many kinds of inhomogeneity shapes by changing its aspect ratios, such as fibers, penny shape of cracks, slit-like cracks, spheroids, and spheres [22, 39], the proposed inclusion based BEM formulation can be used to study the interaction among these types of inhomogeneities or voids or cracks. In this paper, because the particles are spheres with similar size, as the first step to demonstrate this method, the paper focuses on a solid containing spherical particles, so that the orientation of particles are not considered, i.e. all local coordinates can share the same orientation as the global coordinate. Extension of this method to general multi-physical problems is underway.

\subsection{Numerical implementation and verification}

The above formulation has been implemented in a $\mathrm{C}++$ package - iBEM, which stands for a BEM code addressing inhomogeneity or inclusion problems. The programming structure is presented in Fig.6, in which each step is elaborated as follows:

1) Given a solid containing a certain number of inhomogeneities in the matrix with perfectly bonded interfaces, the external surface is discretized into boundary elements. This step can be 
treated as a preprocessing. Since only surface is meshed. The number of elements will be reduced significantly compared with the finite element method (FEM) or the general BEM.

2) Go through all the collection points on the surface to calculate individual element matrix for each collection point based on basic boundary element integration. This step is to complete the boundary element formulation. Therefore, the effect of boundary will be considered in this step.

3) Go through each inhomogeneity to build up stress equivalent into global matrix. This is the key step of this proposed iBEM algorithm. Not only the interaction between particles but also the effect from boundary will be taken into account through Eqs.(32) and (33). Notice that if higher order terms of eigenstrain are considered, more equations for each inhomogeneity are required. completeness of the equations can be achieved. The number of unknown for each inhomogeneity with linear terms of eigenstrain is 24 and the unknown for each boundary node is 3 . Therefore the total unknowns are $24 \times$ number of inhomogeneity $3 \times$ number of boundary nodes. The traditional BEM will provide 3 equations for each node, which makes $3 \times$ number of boundary nodes equations. In addition, the equivalent of stress will also provide $24 \times$ number of inhomogeneity equation for the whole system. In the present method, the numbers of equations and unknows are the same. Therefore a closed linear equations system can be formed for numerical solution. If higher order terms of eigenstrain is used, more unknowns in each particle can be used but the same number of equations will also been obtained.

4) Solve the global linear equation system to obtain unknown boundary response and unknown eigensrain. The full LU decomposition [43] with the pivot method has been implemented to calculate the numerical results from the linear equation system .

5) Use the eigenstrain and boundary traction/displacement to calculate the elastic fields, including the stress, strain and displacement fields for analysis. This step can be treated as a postprocessing. As the unknown boundary response and eigenstrains are all obtained, from Eq. (25), one can get the displacement at any field point; therefore from Eq. (26) the inner strain can also be calculated.

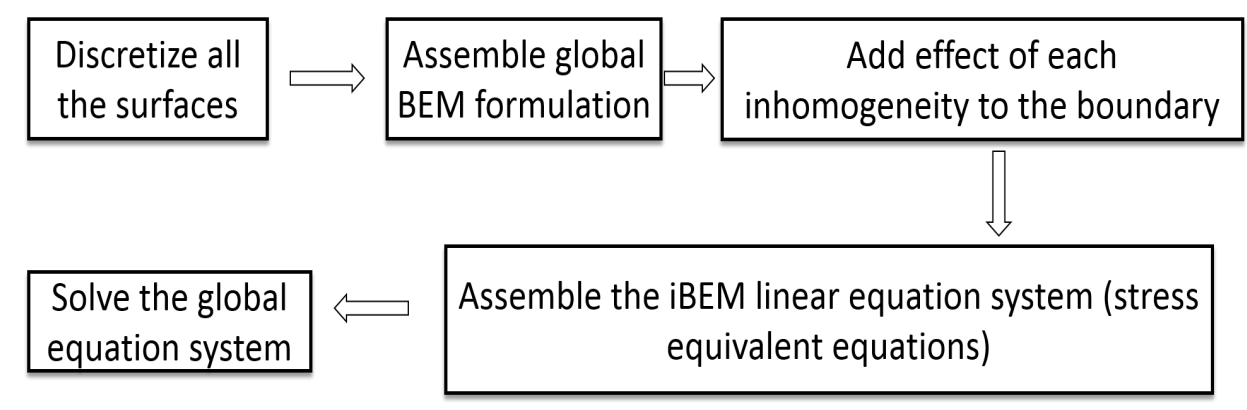

Figure 6: Implementation flow of the iBEM - a software package introducing inhomogeneity/inclusion problems into the BEM code

In order to verify the proposed method, a case study is conducted to investigate two particles located in a simulation box. A cylinder model is built up with a diameter of 1 and a height of 2 . 
Two voids are located in the cylinder. The diameter of both voids are 0.4 . One is centered at the position $(0,0,0.65)$. The another is centered at the position $(0,0,1.35)$. The Young's modulus is $16.9 \mathrm{GPa}$ and Poisson's Ratio is 0.25 . The cylinder is fixed in all directions at the bottom and subjected to a uniform compressive load of $10 \mathrm{MPa}$ at the top. The configuration is shown in Fig.7. A model of this cylinder is also generated in ABAQUS for finite element simulation of an axisymmetric problem with 10570 elements. The mesh is highly refined to get high accuracy . In the iBEM, only a mesh for the surface of cylinder is used, so that 1423 elements are used. Notice that ABAQUS uses a 2D mesh whereas iBEM uses a 3D mesh with a much lower number. Since a linear shape function is used here to interpolate the curved surface, errors in the boundary geometry can be incurred but they will be reduced with more elements used.

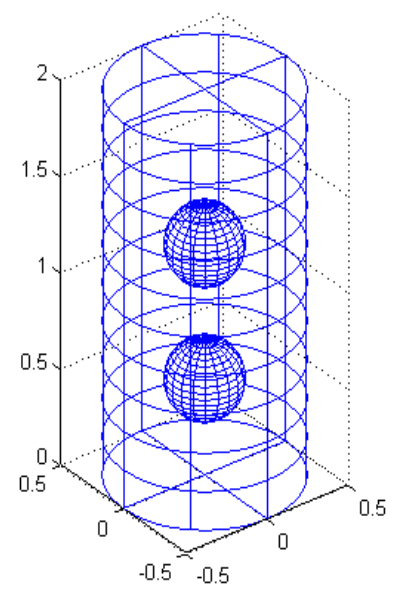

(a)
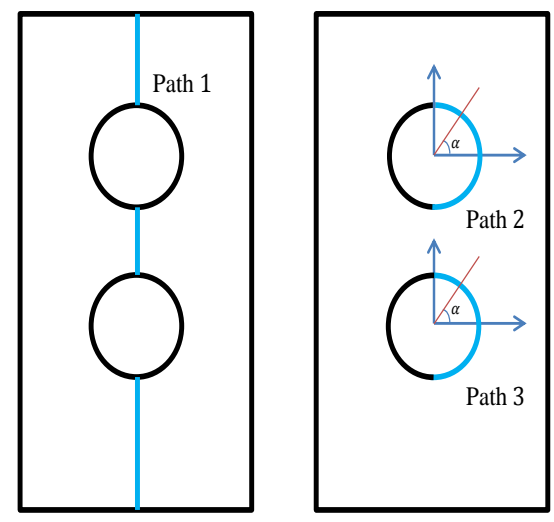

(b)

Figure 7: Configuration of the simulation model -(a) 3D geometry and (b) the path to show the points of interest

The elastic field distributed along three lines are selected to make comparison with the FEM results. For the ease of description, both Cartesian coordinate and cylindrical coordinate notations will be used with $\mathrm{x}, \mathrm{y}, \mathrm{z}$ and $\mathrm{r}, \theta, \mathrm{z}$ in consistent with the literature. Here, $\mathrm{x}, \mathrm{y}$, and $\mathrm{z}$ are the same as the index form of $x_{1}, x_{2}$, and $x_{3}$ in the derivation, respectively. The first line is starting from $(0,0,0)$ along with $\mathrm{z}$ direction to $(0,0,2)$. All points share the same $\mathrm{x}$ and $\mathrm{y}$ coordinates but with a varying $\mathrm{z}$ coordinates. The second and third lines are looped around both voids. The angle in the comparison figure is from the local polar coordinate of each void. The configuration of the simulation model is shown as Fig.7. Some results using iBEM with linear eigenstrain distributions are shown in Figs.8-13.

Based on comparison of the displacements, very good agreement between FEM and iBEM is observed. In Fig.8, as the coordinate $\mathrm{z}$ increases, the displacement in the $\mathrm{z}$ direction also increases. The gap between 0.45 and $0.85,1.15$ and 1.55 are because of presence of voids. Figs.9(a) and 10(a) show that displacements $u_{x}$ are symmetric to the $\mathrm{z}$ axis, due to this being an axisymmetric problem about the $\mathrm{z}$ axis. The displacement comparison in Figs.9(b) and 10(b) shows that due to an increase 


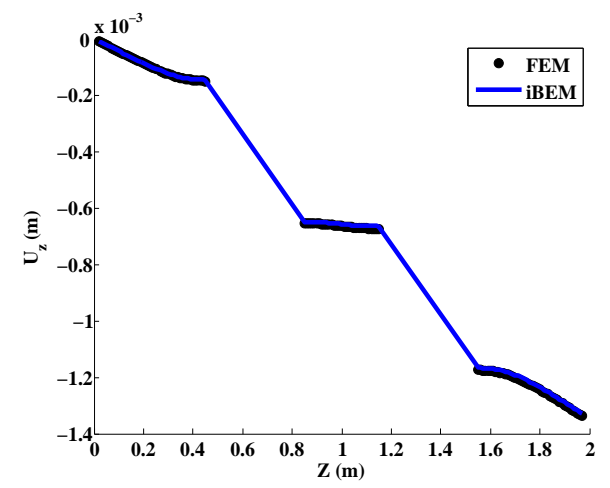

Figure 8: Comparison of displacement between the FEM and iBEM results along Path One.

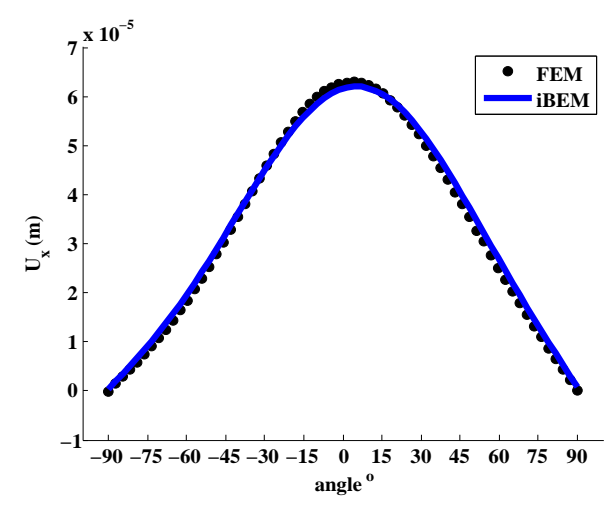

(a)

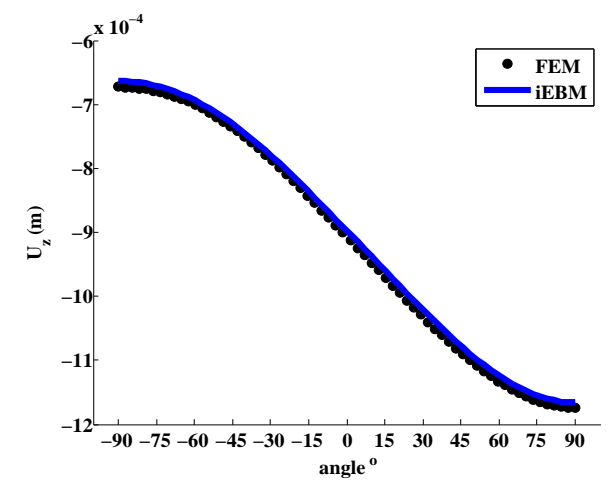

(b)

Figure 9: Comparison of displacement in (a) the $\mathrm{x}$ direction and (b) the $\mathrm{z}$ direction between FEM and iBEM results along Path Two

of the $\mathrm{z}$ coordinate, the displacement in the $\mathrm{z}$ direction also increases. The displacement in the $\mathrm{x}$ direction for each hole is relatively small compared with the displacement in the $\mathrm{z}$ direction, this is because our loading condition is purely compression. Also the maximum displacement in the $\mathrm{x}$ direction for each hole appears at the point where the $\mathrm{x}$ coordinate is at the maximum value, this is due to the effect of Poisson's ratio.

Fig.11 illustrates that the stress $\sigma_{z z}$ will approach $10 \mathrm{MPa}$ as the z coordinate increases, which satisfies the boundary condition at the loading end.. However, in Fig.11, considerable differences in the stress exists in the neighborhood of the void. When higher order terms are considered, more accurate results are expected. Even though only up to linear terms are considered, the present model can still provide fairly good prediction of the stress distribution. Figs.12-13 demonstrate the stress around the top and bottom voids. Since it is a traction free surface around the void, only stress $\sigma_{\theta \theta}$ exists, which maximizes at $\theta=0$ due to stress concentration. When $\theta$ is changed far 


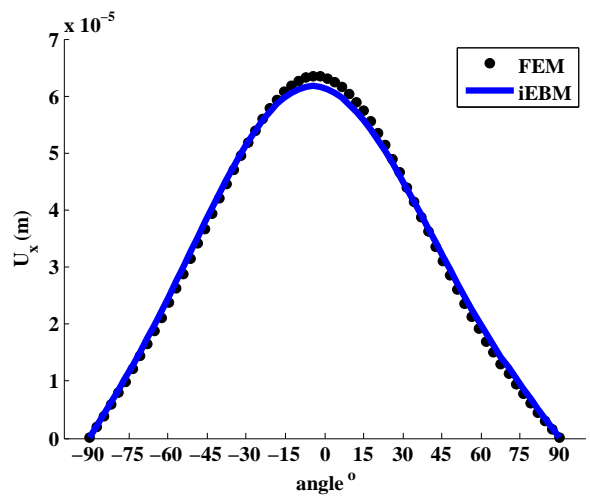

(a)

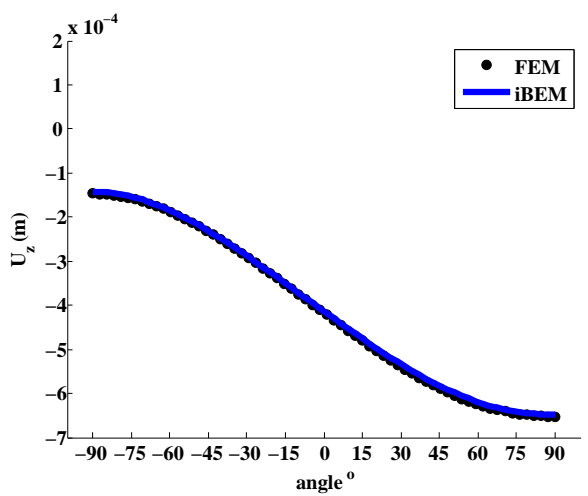

(b)

Figure 10: Comparison of displacement in (a) the $\mathrm{x}$ direction and (b) the $\mathrm{z}$ direction between the FEM and iBEM results along Path Three

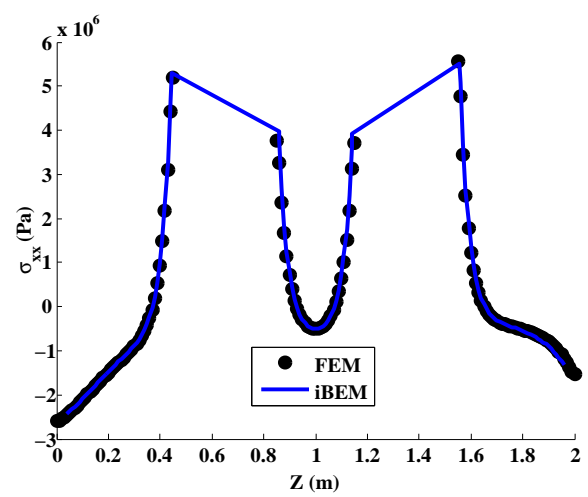

(a)

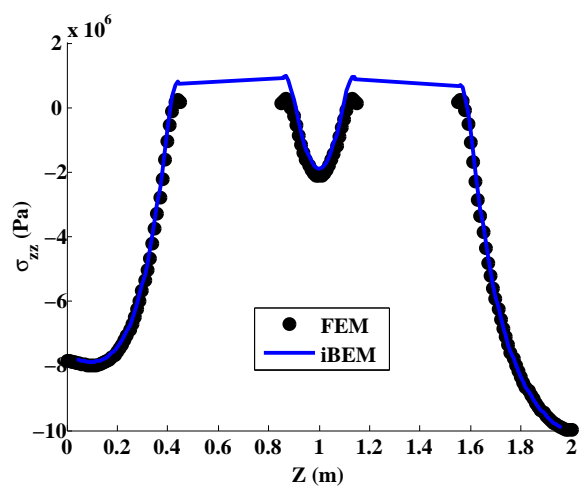

(b)

Figure 11: Stress comparison (a) $\sigma_{x x}$ and (b) $\sigma_{z z}$ between FEM and iBEM results along Path One

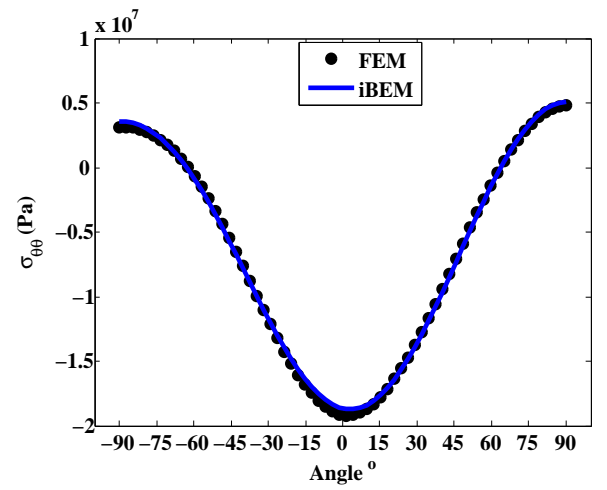

Figure 12: Stress comparison $\sigma_{\theta \theta}$ between the FEM and iBEM results along Path Two 


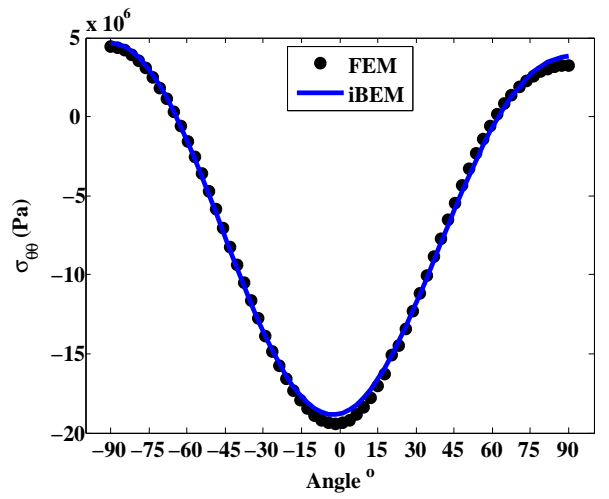

Figure 13: Stress comparison $\sigma_{\theta \theta}$ between the FEM and iBEM results along Path Three

away from 0 , the stress $\sigma_{\theta \theta}$ decreases to zero first and then reaches positive values, which indicates that the top and bottom of each void is under tension, which may lead to a different failure mode and will be discussed in the last Section.

\subsection{Asymptotic analysis}

The above studies have used the Taylor expansion of eigenstrain up to the linear terms. Although very good agreement has been demonstrated between the iBEM and FEM results, the accuracy of the solution may change case by case, especially when the particle is close to the boundary or two particles are very close to each other. Using higher order terms of eigenstrain will be able to reach higher accuracy. In this section, the asymptotic analysis is conducted with the eigenstrain in Eq. (23) up to quadratic terms.

Based on Mura's previous work[22], the quadratic term in equivalent inclusion method plays an significant role when the particle-particle distance is small. In the following asymptotic analysis, an example is built with the following configuration including two particles which is similar to the example of Fig.7 in Subsection 3.5 but with a much smaller spacing of 0.1. A cylinder model is built up with a diameter of 1 and a height of 2 . Two voids are located in the cylinder. The diameter of both voids are 0.4 . One is located at the position $(0,0,0.75)$. The another is located at the position $(0,0,1.25)$. The Young's modulus is $1 \mathrm{MPa}$. Poisson's Ratio is 0.25 . The cylinder is subjected to a uniform compressive load of $0.04 \mathrm{MPa}$ at the top and is fixed at the bottom. An axisymmetric FEM model of this cylinder is also generated in ABAQUS. The mesh is highly refined with 11201 elements to assure high accuracy. In the iBEM part, the same mesh of the surface of cylinder is also generated as Fig.7 in Subsection 3.5 with 1474 elements in total. Using constant, linear, and quadratic form of eigenstrain field on the inhomogeneities, the stress distributions along Path One, Path Two and Path Three are illustrated as the following.

Figs.14-16 show that the higher order form of eigenstrain distribution provides higher accuracy in general when the particles are close to each other. The quadratic case provides the best agreement with the FEM results because it enables the best capacity to fit the stress distribution on the 


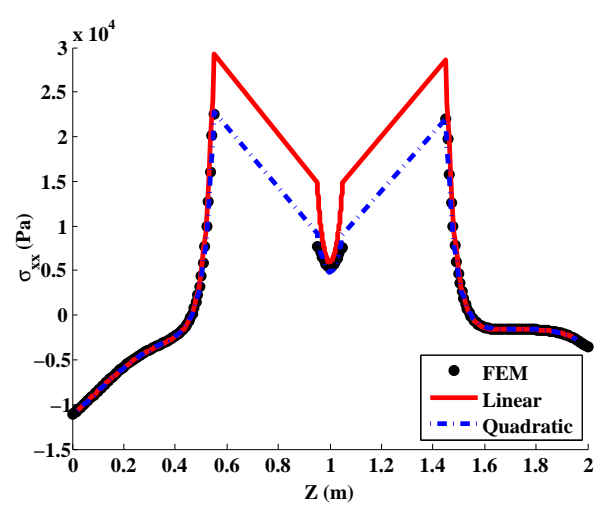

(a)

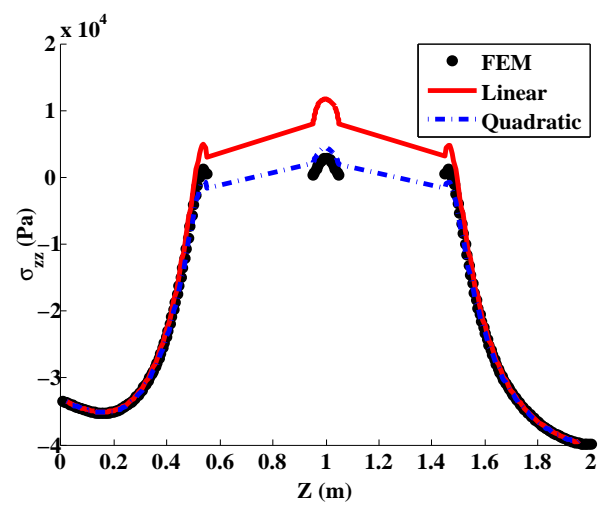

(b)

Figure 14: Stress comparison (a) $\sigma_{x x}$ and (b) $\sigma_{z z}$ between the FEM and iBEM both linear and quadratic results along Path One

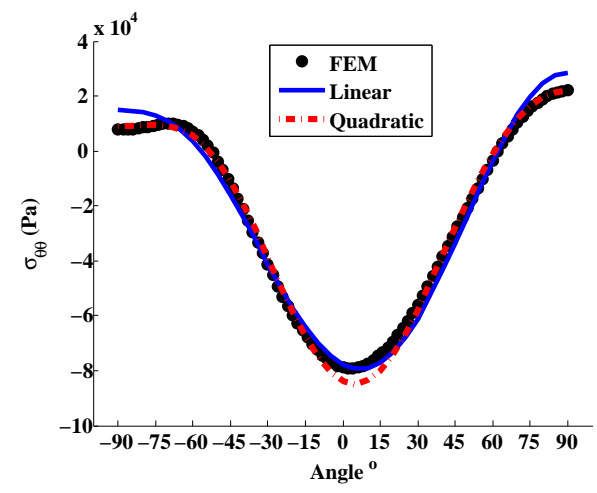

Figure 15: Stress comparison $\sigma_{\theta \theta}$ between the FEM and iBEM both linear and quadratic results along Path Two

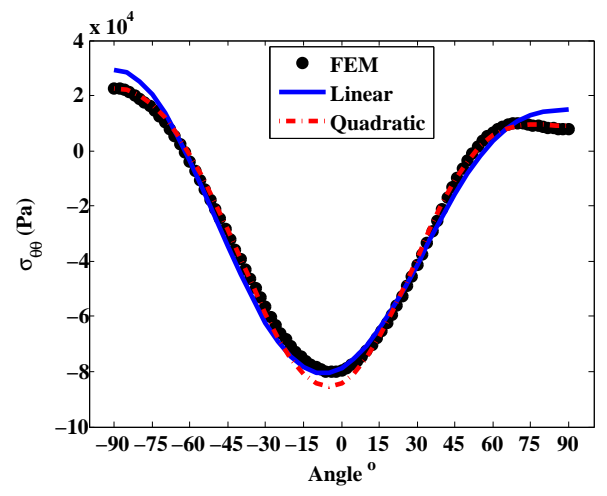

Figure 16: Stress comparison $\sigma_{\theta \theta}$ between the FEM and iBEM both linear and quadratic results along Path Three 
inhomogeneities. However, when the particles are far away, the difference among the three cases will be reduced because the strain distribution becomes more uniform. The effective of quadratic eigenstrain term will fade away significantly respect to the distance between particles. If enough terms are considered in the Taylor expansion on both eigenstrain and total strain, the numerical results may approach the exact solution.

\section{Results and Discussion}

In general, Eshelby's equivalent inclusion method (EIM) addresses the material inhomogeneity problem by replacing the inhomogeneity with matrix material and introducing a non-mechanical strain (eigenstrain) to make the constitutive law of Eq. (31) equivalently satisfied. Therefore, the boundary value problem (BVP) is transferred to a homogeneous solid with an eigenstrain in the particle domains, which can be analytically solved with elliptic integrals for ellipsoidal particles. Compared with the boundary integral method (BIM), the present method uses eigenstrain to represent the material mismatch so that it does not need to mesh the particle interface into elements. For the single inhomogeneity case, the BEM may handle with this problem very well if the smoothly curved surface is carefully addressed. However, when the number of particles approaches 10, 50 or 100 , and if the particle size changes in a large range, it is formidable to use the BEM, but the present method works very well as shown in another recent paper [40]. This section will demonstrate the present method and compare the modeling results with the experiments. Although this model can handle problems with various microstructures at the same level of computational cost, for simplicity and clear quantification, we use a simple cubic lattice microstructure to idealize the particle distribution.

\subsection{Effective elastic moduli}

In order to get the effective moduli of the LWC, a cubic unit cell is generated with a unit length for each edge. The Young's modulus and Poisson's ratio of the matrix are $E=16.9 \mathrm{GPa}$ and $v=0.25$ which are obtained from our samples of pure cement paste without polymer foam particles. Since polymer foam particles in the concrete cannot bear any load and they are mainly used to reduce the density of concrete, they can be treated as inhomogeneity with zero stiffness. In other words, they are equivalent to voids with $E=0 \mathrm{GPa}$ embedded in the cement matrix. Because the two ends of a cylinder specimen are capped with a flat surface, the top and bottom surfaces are assumed to remain flat during the test. Therefore in our simulation, a uniform displacement $\left(u_{z}=0.05, u_{x}=u_{y}=0\right)$ is applied on the top surface and the bottom is fixed at all directions $\left(u_{x}=u_{y}=u_{z}=0\right)$. For other surfaces, they are all traction free. A certain number of particles, say 1,8 , and 27 particles respectively, are uniformly distributed in this domain in a simple cubic lattice. By changing the diameter of particles, the different volume fraction can be represented. The effective stiffness will be calculated by the following procedure.

The effective elasticity of the composites correlate the effective strain $\langle\boldsymbol{\epsilon}\rangle$ and stress $\langle\boldsymbol{\sigma}\rangle$ by the following equation: 


$$
\left\langle\sigma_{i j}\right\rangle=\left\langle C_{i j k l}\right\rangle:\left\langle\epsilon_{k l}\right\rangle
$$

where the effective strain can be derived by the boundary integral of a continuous displacement field as follows

$$
\left\langle\epsilon_{i j}\right\rangle=\frac{1}{V} \int_{D} \epsilon_{i j} d \mathbf{x}=\frac{1}{2 V} \int_{D}\left(u_{i, j}+u_{j, i}\right) d \mathbf{x}=\frac{1}{2 V} \int_{\partial D}\left(u_{i} n_{j}+u_{j} n_{i}\right) d \mathbf{x} .
$$

For the loading condition in the experiments, the effective strain in the loading direction $(z$ direction) can be written as.

$$
\left\langle\epsilon_{33}\right\rangle=\frac{1}{V} \int_{\partial D} u_{3} n_{3} d \mathbf{x}
$$

where only the top and bottom surface contributes to the integral. By approximation, we will average the displacement on the top and bot surface to get $\langle\epsilon\rangle_{33}$ as

$$
\left\langle\epsilon_{33}\right\rangle=\frac{1}{h}\left(\bar{u}_{3}^{t o p}-\bar{u}_{3}^{b o t}\right)
$$

in which based on our loading condition, $\bar{u}_{3}^{\text {top }}=0.05, \bar{u}_{3}^{\text {bot }}=0$ and $h=1$, the effective strain can be calculated straightforwardly.

The average stress can also be written in term of the traction on the boundary as

$$
\begin{aligned}
\left\langle\sigma_{i j}\right\rangle & =\frac{1}{V} \int_{D} \sigma_{i j} d \mathbf{x} \\
& =\frac{1}{V} \int_{D}\left[\left(\sigma_{i k} x_{j}\right)_{, k}-\sigma_{i k, k} x_{j}\right] d \mathbf{x}=\frac{1}{V} \int_{\partial D}\left(\sigma_{i k} n_{k} x_{j}\right) d \mathbf{x}=\frac{1}{V} \int_{\partial D}\left(t_{i} x_{j}\right) d \mathbf{x}
\end{aligned}
$$

Similarly, only the top and bottom contribute to the above integral. Therefore, the average stress $\left\langle\sigma_{33}\right\rangle$ can be written as the surface integral of traction in the $z$ direction on the top surface, i.e.

$$
\left\langle\sigma_{33}\right\rangle=\bar{t}_{3}^{t o p}
$$

Finally the effective Young's modulus can be expressed as

$$
E=\left\langle\sigma_{33}\right\rangle /\left\langle\epsilon_{33}\right\rangle
$$

In order to show the effect of the number of particles in one unit cell, 1, 8, and 27 particles are considered and the effective Young's moduli are illustrated in Fig.17. When one particle is considered, the result is higher than those for the cases of 8 particles and 27 particles. This is reasonable because the particle interaction tends to intensify the effect of particles, which leads to a lower Young's modulus. However, the cases of 8 particles and 27 particles present very close results, which means that 8 particles are enough to provide a reliable prediction of effective Young's 


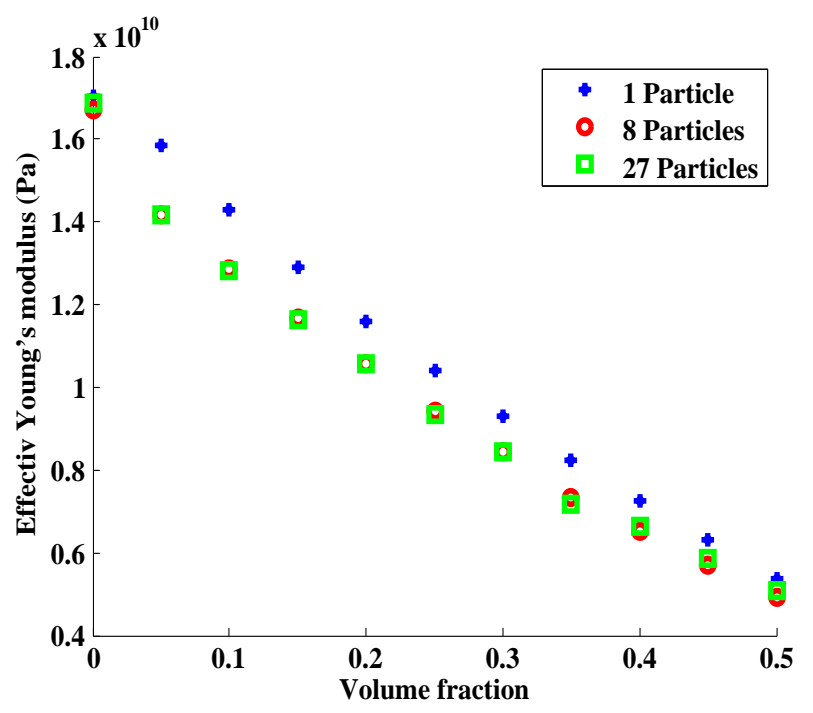

Figure 17: The effective Young's modulus based on different number of particles

modulus for a composite sample, which will be used in the following comparisons. Notice that the size effect is a very important factor for the real situation and experiments where imperfect interfaces or interface layers exist, which is a new physics that this paper did not study. The above statement about convergence is based on the assumption of linear elastic inhomogeneity with perfect bounding considering the boundary effect and particle?s interaction. Under this condition, only volume fraction and boundary effects play roles in the effective material properties and the size effect of particles is not considered.

Fig.18 shows the experimental results of effective Young's modulus for LWC samples containing different volume fractions of foam particles as well as the predictions of the Mori-Tanaka model, the self-consistent model, the Voigt model, the dilute model [23] and the present iBEM prediction. In the figure, the Voigt and dilute models can not predict the effective Young's modulus of LWC very well. The present modeling result is close to the Mori-Tanaka model but exhibits a better agreement with the experimental results. The self-consistent model provides similar prediction to other models when the volume fractions of particles are small but then directly approach zero when the volume fraction is 0.5 , which is not physical. For the porous materials, the lower bound models, such as the Ruess model and the Hashin-Shtrikman lower bound, provide a prediction of zero stiffness, which is not physical either, so we do not show it.

Notice that the analytical micromechanics-based models cannot actually consider the effect of microstructure, but the present model can consider a large number of particles with idealized particle distributions. This also demonstrates the capability of our method to homogenize the mechanical properties of composites with different volume fractions. Notice that although the present iBEM model only studies the microstructure of the simple cubic lattice, it provides very good predictions to the experimental results. If an actual microstructure is given, the iBEM code 
can straightforwardly predict the elastic behavior in the same fashion.

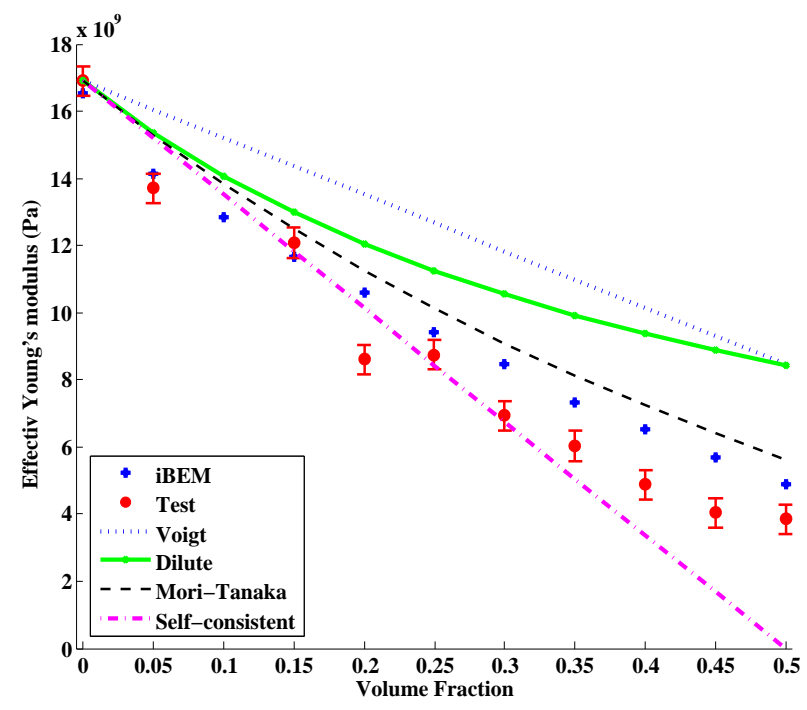

Figure 18: Relation between Young's modulus and volume fraction

\subsection{Effective strength}

The effective strength of the lightweight concrete is a key parameter in specific structural design. From the experimental results, adding foam particles will significantly decrease the strength of the lightweight concrete. Concrete is very brittle. A crack is hard to initiate in concrete when it is subjected to compressive stress conditions but easy to initiate when tensile stress exists. This results in the compressive strength of concrete being is several times higher than the tensile strength. In lightweight concrete, there exists interfaces between the cement and each particle, which may change the stress distribution inside the concrete significantly when it is subjected to pure compressive load. Without the particles, all the domain inside the concrete experiences a condition of pure compressive stress, with the value is equal to the far field stress. However, after adding soft foam particles, the hoop stress around the interface can not only be negative but also positive. It varies from compressive stress to tensile stress as $\alpha$ changing from 0 to 90 as shown in Fig.19.Also as the volume fraction is increased, the maximum concentrated compressive and tensile stress also increase. This is the reason why the strength of lightweight concrete comprised of foam particles will significantly decrease even though the lightweight concrete is subjected to a pure compressive load.

Our criterion for the failure is governed by the maximum hoop stress (tensile or compressive) around the void which is compared with the critical tensile or compressive stress. This section is an empirical study based on the experiment data. We transfer the load to the maximum hoop stress (tensile or compressive) around the voids based on the ratio as shown in Table 3 which indicates the effect of volume fraction. Based on the volume fraction of 0 and 5 percentage, we can determine 


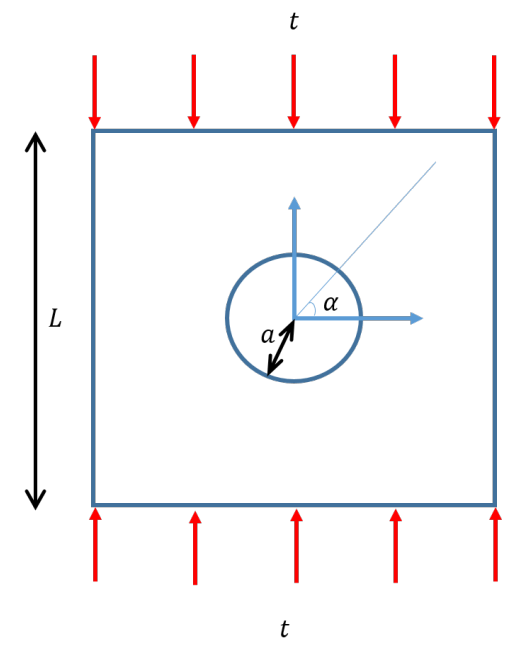

Figure 19: Configuration of one unit cell

the critical compressive stress and critical tensile stress. Our analysis does not include the plastic and damage process. It is a simple and empirical analysis, but it predicted the strength of the lightweight concrete very well. Further experimental and numerical investigation is still underway.

To study the effect of local stress on the effective strength in a lightweight concrete, as the first step, we simplify this complex problem into a single particle case. Here, we investigate the maximum compressive hoop stress and maximum tensile hoop stress in one unit cell containing one particle in Fig.19 and use them to determine the failure initiation. By changing the ratio of $a$ to $L$, we can equivalently study the effect of different volume fractions.

The ratios of maximum tensile hoop stress and maximum compressive hoop stress to the load $t$ for different volume fractions are recorded as shown in Table 3. Based on the first experiment data with volume fraction of zero, the critical compressive yield stress can be obtained as 7,027 psi. Also based on the ratio for a volume fraction of 0.05 , the critical tensile yield stress is 4,127 psi. Using them as the compressive and tensile strengths, for different volume fractions of foam particles, we can compare the maximum compressive or tensile stress with their strengths to determine the critical load $t$ as the effective strength of the LWC. Therefore, the predicted strengths based on critical stress and the corresponding ratio can be calculated. The comparison is shown in Fig.20.

The predicted results are in good agreement with the test results as shown in Fig.20. The results show that the failure criteria for low volume fractions is governed by that tensile stress, but this is not the case for volume fractions greater than and equal to 0.3. Notice that the strength of the LWC changes significantly with different microstructures. The purpose of the current iBEM model is to study the overall trend of the strength changing with the volume fractions of foam particles. The present model with a single particle only qualitatively demonstrates the effect of the local stress on the effective strength of LWC. The variability of test results and particle interaction on the strength of LWC will be studied in our future work. 
Table 3: The absolute value of the ratio of maxmimum compressive stress and tensile stress to the load $t$

\begin{tabular}{ccc}
\hline Volume fraction(\%) & $\begin{array}{c}\text { Ratio of maximum tensile hoop } \\
\text { stress to load } t\end{array}$ & $\begin{array}{c}\text { Ratio of maximum compressive hoop } \\
\text { stress to load } t\end{array}$ \\
\hline 0 & 0.0 & 1.0 \\
5 & 0.728 & 2.287 \\
10 & 0.934 & 2.635 \\
15 & 1.132 & 2.997 \\
20 & 1.308 & 3.381 \\
25 & 1.428 & 3.793 \\
30 & 1.462 & 4.266 \\
35 & 1.370 & 4.872 \\
40 & 1.060 & 5.767 \\
45 & 0.0 & 7.362 \\
50 & 0.0 & 12.832
\end{tabular}

\section{Conclusions}

This paper investigates the effective mechanical property of a novel lightweight concrete containing different volume fractions of foam particles in the both experimental and theoretical perspectives.The test results show that as the volume fraction of polymer increases, the strength and Young's modulus decreases. It means the density can reflect the overall quality of this lightweight concrete. By linear regression, the decrease of strength is approximately 96 psi per a 0.01 increase in the volume fraction, and the decrease of Young's modulus is $0.26 \mathrm{GPa}$ per a 0.01 increase in the volume fraction. The Poisson's ratio does not show a certain trend and it oscillates around 0.3. A new equivalent inclusion based boundary element method (iBEM) is presented which can deal with a large number of inhomogeneities embedded in composite materials efficiently for 3-D problems. The effect of the boundary and particle interactions on the local stress field and overall effective elastic moduli can be taken into account. The method is verified with the finite element method in ABAQUS. An asymptotic analysis reveals that higher accuracy can be achieved if we increase the terms in the Taylor expansion of the eigenstrain.

Numerical homogenization is conducted to predict the effective Young's modulus. The difference in the Young's modulus of unit cells containing 1,8 and 27 particles indicates that the interaction between particles tends to intensify the effect of particles, which ultimately leads to a lower Young's modulus than if the interaction did not exist. However, the cases of 8 particles and 27 particles present very close results, which means that additional particles may not provide significant contribution any more. The comparison of the iBEM results with other conventional micromechanical models shows that the present iBEM provides better predictions especially for high volume fractions of particles. The strength of the lightweight concrete is studied with a unit cell containing a single particle. Using the maximum tensile stress and compressive stress around the interface of the void, we can determine if the structure fails by tensile stress or compressive 


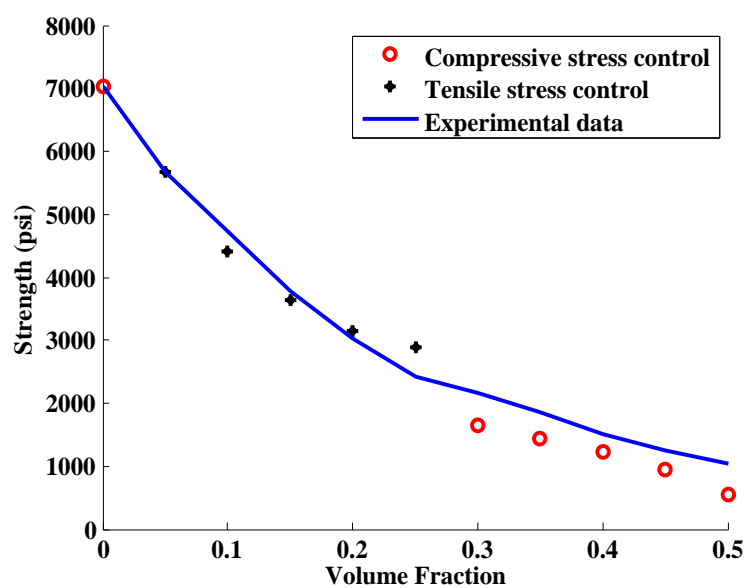

Figure 20: Relation between strength and volume fractions

stress. The result shows that the failure of the composites is governed by tensile stress for a low volume fraction of particles, but by compressive stress for a volume fraction greater than or equal to 0.3. Although the microstructure of LWC has been idealized with unit cells with a few particles, the iBEM modeling results can predict the elastic moduli and strengths with acceptable accuracy.

The strength analysis for lightweight concrete does not include any plastic and damage process. More efforts for establishing rigorous framework and nonlinear analysis in the strength analysis of lightweight concrete should be taken in the future. The simulation with actual particle distributions is underway. The iBEM program can be used in virtual experiments for mechanical behavior of particulate composites.

\section{Acknowledgements}

The authors acknowledge the financial support from National Science Foundation CMMI 0954717, CMMI 1301288, and CMMI 1301160. The authors are also grateful to Professor C. Meyer, Dr. J.Q. Wei, Dr. D. Hochstein, and Dr. L.M. Li for some valuable information about the material preparation and experimental tests. We are also grateful to Professor Y.S. Chan, J.S. Chen and Dr. D. Hochstein for some valuable discussion and manuscript preparation. In addition, author Yin appreciates the support of the Henry Mitchell Weitzner Research Fund, which has been and will be used in his research of roofing materials for solar energy applications and technologies. The results and opinions presented herein are those of the authors and do not necessarily reflect those of the sponsoring agency. 


\section{Appendix}

A Expression of the integral terms of $\Phi, \Psi, \Phi_{p}$, and $\Psi_{p}$ and their derivatives

Following the concept from Mura [22] and Yin [42], $\Phi$ and $\Psi$ denote the integral of the functions $\phi$ and $\psi$ over an ellipsoidal inclusion $\Omega$ as

$$
\Phi=\int_{\Omega} \phi d x^{\prime}, \text { and } \Psi=\int_{\Omega} \psi d x^{\prime}
$$

where $\phi$ and $\psi$ are defined in Eq. (21). In the following, $\Phi_{p}$ and $\Psi_{p}$ denote the integral of $\phi x_{p}^{\prime}$ and $\psi x_{p}^{\prime}$

The integral terms of $\Phi, \Psi, \Phi_{p}$, and $\Psi_{p}$ and their derivative terms over an ellipsoidal domain can be derived and expressed as

$$
\begin{aligned}
& \Phi=\frac{1}{2}\left[I(\lambda)-x_{r} x_{r} I_{R}(\lambda)\right] \\
& \Phi_{, i}=-x_{i} I_{I}(\lambda) \\
& \Phi_{, i j}=-\delta_{i j} I_{I}(\lambda)-x_{i} I_{I, j} \\
& \Phi_{p}=\frac{1}{2} a_{p}^{2} x_{p}\left[I_{p}(\lambda)-x_{r} x_{r} I_{R P}(\lambda)\right] \\
& \Phi_{p, i}=\frac{1}{2} a_{P}^{2}\left\{\delta_{i p}\left[I_{P}(\lambda)-x_{r} x_{r} I_{R P}(\lambda)\right]-2 x_{p}\left[x_{i} I_{I P}(\lambda)\right]\right\} \\
& \Phi_{p, i j}=-a_{P}^{2}\left[\delta_{i p} x_{j} I_{J P}(\lambda)+\delta_{j p} x_{i} I_{I P}(\lambda)+\delta_{i j} x_{p} I_{I P}(\lambda)+x_{i} x_{p} I_{I P, j}(\lambda)\right] \\
& \Psi_{, i j}=\frac{1}{2} \delta_{i j}\left[I(\lambda)-x_{r} x_{r} I_{R}(\lambda)\right]-\frac{1}{2} \delta_{i j} a_{I}^{2}\left[I_{I}(\lambda)-x_{r} x_{r} I_{R I}(\lambda)\right]-x_{i} x_{j}\left[I_{J}(\lambda)-a_{I}^{2} I_{I J}(\lambda)\right] \\
& \Psi_{, i j k}=-\delta_{i j} x_{k}\left[I_{K}(\lambda)-a_{I}^{2} I_{I K}(\lambda)\right]-\left(\delta_{i k} x_{j}+\delta_{j k} x_{i}\right)\left[I_{J}(\lambda)-a_{I}^{2} I_{I J}(\lambda)\right]-x_{i} x_{j}\left[I_{J}(\lambda)-a_{I}^{2} I_{I J}(\lambda)\right]_{, k} \\
& \Psi_{, i j k l}=\left\{\begin{array}{c}
-\delta_{i j} \delta_{k l}\left[I_{K}(\lambda)-a_{I}^{2} I_{I K}(\lambda)\right]-\delta_{i j} x_{k}\left[I_{K}(\lambda)-a_{I}^{2} I_{I K}(\lambda)\right]_{, l} \\
-\left(\delta_{i k} \delta_{j l}+\delta_{j k} \delta_{i l}\right)\left[I_{J}(\lambda)-a_{I}^{2} I_{I J}(\lambda)\right]-\left(\delta_{i k} x_{j}+\delta_{j k} x_{i}\right)\left[I_{J}(\lambda)-a_{I}^{2} I_{I J}(\lambda)\right]_{, l} \\
-\left(\delta_{i l} x_{j}+\delta_{j l} x_{i}\right)\left[I_{J}(\lambda)-a_{I}^{2} I_{I J}(\lambda)\right]_{, k}-x_{i} x_{j}\left[I_{J}(\lambda)-a_{I}^{2} I_{I J}(\lambda)\right]_{, k l}
\end{array}\right\} \\
& \Psi_{p, i j}=\frac{1}{2} a_{P}^{2}\left\{\begin{array}{c}
\delta_{i p} x_{j}\left[I_{J}(\lambda)-x_{r} x_{r} I_{R J}(\lambda)\right]-\delta_{i p} x_{j} a_{P}^{2}\left[I_{J P}(\lambda)-x_{r} x_{r} I_{R J P}(\lambda)\right] \\
+\left(\delta_{j p} x_{i}+\delta_{i j} x_{p}\right)\left[I_{I}(\lambda)-x_{r} x_{r} I_{R I}(\lambda)\right] \\
-a_{P}^{2}\left(\delta_{j p} x_{i}+\delta_{i j} x_{p}\right)\left[I_{I P}(\lambda)-x_{r} x_{r} I_{R I P}(\lambda)\right]+2 x_{i} x_{j} x_{p}\left(-I_{I J}+a_{P}^{2} I_{I J P}\right)
\end{array}\right\}
\end{aligned}
$$




$$
\begin{aligned}
& \Psi_{p, i j k}=a_{P}^{2}\left\{\begin{array}{c}
\frac{1}{2} \delta_{i p} \delta_{j k}\left[I_{J}(\lambda)-x_{r} x_{r} I_{R J}(\lambda)\right] \\
-\frac{1}{2} \delta_{i p} \delta_{j k} a_{P}^{2}\left[I_{J P}(\lambda)-x_{r} x_{r} I_{R J P}(\lambda)\right] \\
+\delta_{i p} x_{j}\left[-x_{k} I_{K J}(\lambda)+a_{P}^{2} x_{k} I_{K J P}(\lambda)\right] \\
+\frac{1}{2}\left(\delta_{j p} \delta_{i k}+\delta_{i j} \delta_{k p}\right)\left[I_{I}(\lambda)-x_{r} x_{r} I_{R I}(\lambda)\right] \\
-\frac{1}{2}\left(\delta_{j p} \delta_{i k}+\delta_{i j} \delta_{k p}\right) a_{P}^{2}\left[I_{I P}(\lambda)-x_{r} x_{r} I_{R I P}(\lambda)\right] \\
+\left(\delta_{j p} x_{i}+\delta_{i j} x_{p}\right)\left[-x_{k} I_{K I}(\lambda)+a_{P}^{2} x_{k} I_{K J P}(\lambda)\right] \\
+\left(\delta_{i k} x_{j} x_{p}+\delta_{j k} x_{i} x_{p}+\delta_{k p} x_{i} x_{j}\right)\left[-I_{I J}(\lambda)+a_{P}^{2} I_{I J P}(\lambda)\right] \\
+x_{i} x_{j} x_{p}\left[-I_{I J}(\lambda)+a_{P}^{2} I_{I J P}(\lambda)\right]_{, k}
\end{array}\right\} \\
& \Psi_{p, i j k l}=a_{P}^{2}\left\{\begin{array}{c}
\delta_{i p} \delta_{j k} x_{l}\left(-I_{L J}+a_{P}^{2} I_{L J P}\right)+\left(\delta_{i p} \delta_{j l} x_{k}+\delta_{i p} \delta_{k l} x_{j}\right)\left(-I_{K J}+a_{P}^{2} I_{K J P}\right) \\
+\left(\delta_{i j} \delta_{k p} x_{l}+\delta_{i k} \delta_{j p} x_{l}\right)\left(-I_{L I}+a_{P}^{2} I_{L I P}\right) \\
+\left(\delta_{i j} \delta_{k l} x_{p}+\delta_{i j} \delta_{l p} x_{k}+\delta_{i l} \delta_{j p} x_{k}+\delta_{j p} \delta_{k l} x_{i}\right)\left(-I_{K I}+a_{P}^{2} I_{K I P}\right) \\
+\left(\delta_{i k} \delta_{j l} x_{p}+\delta_{i k} \delta_{l p} x_{j}+\delta_{i l} \delta_{j k} x_{p}\right)\left(-I_{J I}+a_{P}^{2} I_{J I P}\right) \\
+\left(\delta_{i l} \delta_{k p} x_{j}+\delta_{j k} \delta_{l p} x_{i}+\delta_{j l} \delta_{k p} x_{i}\right)\left(-I_{J I}+a_{P}^{2} I_{J I P}\right) \\
+\delta_{i p} x_{j} x_{k}\left(-I_{K J}+a_{P}^{2} I_{K J P}\right)_{, l}+\left(\delta_{j p} x_{i} x_{k}+\delta_{i j} x_{k} x_{p}\right)\left(-I_{K I}+a_{P}^{2} I_{K I P}\right)_{, l} \\
+\left(\delta_{i k} x_{j} x_{p}+\delta_{j k} x_{i} x_{p}+\delta_{k p} x_{i} x_{j}\right)\left(-I_{J I}+a_{P}^{2} I_{J I P}\right)_{, l} \\
+\left(\delta_{i l} x_{j} x_{p}+\delta_{j l} x_{i} x_{p}+\delta_{l p} x_{i} x_{j}\right)\left(-I_{J I}+a_{P}^{2} I_{J I P}\right)_{, k} \\
+x_{i} x_{j} x_{p}\left(-I_{J I}+a_{P}^{2} I_{J I P}\right)_{, k l}
\end{array}\right\}
\end{aligned}
$$

where Mura's extended index notation [22] is used, namely, repeated lower case indices are summed up as usual index notation, and; upper case indices take on the same numbers as the corresponding lower case ones but are not summed. In the above equation, the following elliptic integrals are used:

$$
\left\{\begin{array}{l}
I(\lambda)=2 \pi a_{1} a_{2} a_{3} \int_{\lambda}^{\infty} \frac{d s}{\Delta(s)} \\
I_{i}(\lambda)=2 \pi a_{1} a_{2} a_{3} \int_{\lambda}^{\infty} \frac{d s}{\left(a_{i}^{2}+s\right) \Delta(s)} \\
I_{i j}(\lambda)=2 \pi a_{1} a_{2} a_{3} \int_{\lambda}^{\infty} \frac{d s}{\left(a_{i}^{2}+s\right)\left(a_{j}^{2}+s\right) \Delta(s)} \\
\Delta(s)=\sqrt{\left(a_{1}^{2}+s\right)\left(a_{2}^{2}+s\right)\left(a_{3}^{2}+s\right)}
\end{array}\right.
$$

and $\lambda$ is the largest root of the equation

$$
\frac{x_{1}^{2}}{\left(a_{1}^{2}+\lambda\right)}+\frac{x_{2}^{2}}{\left(a_{2}^{2}+\lambda\right)}+\frac{x_{3}^{2}}{\left(a_{3}^{2}+\lambda\right)}=1
$$


For a spherical domain, i.e. $a_{1}=a_{2}=a_{3}=a$, the integral terms of $\Phi, \Psi, \Phi_{p}$, and $\Psi_{p}$ and their derivative terms can be explicitly derived and expressed as

$$
\begin{aligned}
& \Phi= \begin{cases}\frac{4 \pi}{3} \rho a^{2}, & \text { for } x>a \\
2 \pi\left(a^{2}-\frac{1}{3} x^{2}\right), & \text { for } x \leq a\end{cases} \\
& \Phi_{, i}= \begin{cases}-\frac{4 \pi}{3} \rho^{2} a n_{i}, & \text { for } x>a \\
-\frac{4 \pi}{3} x_{i}, & \text { for } x \leq a\end{cases} \\
& \Phi_{, i j}=\left\{\begin{array}{lr}
-\frac{4 \pi}{3} \rho^{3}\left(-\delta_{i j}+3 n_{i} n_{j}\right), & \text { for } x>a \\
-\frac{4 \pi}{3} \delta_{i j}, & \text { for } x \leq a
\end{array}\right. \\
& \Phi_{p}= \begin{cases}\frac{4 \pi}{15} \rho^{2} a^{3} n_{p}, & \text { for } x>a \\
2 \pi\left(\frac{1}{3} a^{2}-\frac{1}{5} x^{2}\right) x_{p}, & \text { for } x \leq a\end{cases} \\
& \Phi_{p, i}=\left\{\begin{array}{lr}
-\frac{4 \pi}{15} \rho^{3} a^{2}\left(-\delta_{i p}+3 n_{i} n_{p}\right), & \text { for } x>a \\
-\frac{2 \pi}{15}\left[-\left(5 a^{2}-3 x^{2}\right) \delta_{i p}+6 x_{i} x_{p}\right], & \text { for } x \leq a
\end{array}\right. \\
& \Phi_{p, i j}=\left\{\begin{array}{lr}
\frac{4 \pi}{5} \rho^{4} a\left(-\delta_{i j} n_{p}-\delta_{i p} n_{j}-\delta_{j p} n_{i}+5 n_{i} n_{j} n_{p}\right), & \text { for } x>a \\
\frac{4 \pi}{5}\left[-\delta_{i j} x_{p}-\delta_{i p} x_{j}-\delta_{j p} x_{i}\right], & \text { for } x \leq a
\end{array}\right. \\
& \Psi_{, i j}=\left\{\begin{array}{lr}
\frac{4 \pi}{15} \rho a^{2}\left[\left(5-\rho^{2}\right) \delta_{i j}+\left(-5+3 \rho^{2}\right) n_{i} n_{j}\right], & \text { for } x>a \\
\frac{4 \pi}{15}\left[\left(5 a^{2}-x^{2}\right) \delta_{i j}-2 x_{i} x_{j}\right], & \text { for } x \leq a
\end{array}\right. \\
& \Psi_{, i j k}=\left\{\begin{array}{lr}
\frac{4 \pi}{15} \rho^{2} a\left[-\left(5-3 \rho^{2}\right)\left(\delta_{i j} n_{k}+\delta_{i k} n_{j}+\delta_{j k} n_{i}\right)+15\left(1-\rho^{2}\right) n_{i} n_{j} n_{k}\right], & \text { for } x>a \\
-\frac{8 \pi}{15}\left(\delta_{i j} x_{k}+\delta_{i k} x_{j}+\delta_{j k} x_{i}\right), & \text { for } x \leq a
\end{array}\right.
\end{aligned}
$$




$$
\begin{aligned}
& \Psi_{, i j k l}=\left\{\begin{array}{cr}
-\left(5-3 \rho^{2}\right)\left(\delta_{i j} \delta_{k l}+\delta_{i k} \delta_{j l}+\delta_{i l} \delta_{j k}\right) \\
+15\left(1-\rho^{2}\right) \delta_{i j} n_{k} n_{l}+15\left(1-\rho^{2}\right) \delta_{k l} n_{i} n_{j} \\
15 \\
+15\left(1-\rho^{2}\right)\left(\delta_{i k} n_{j} n_{l}+\delta_{i l} n_{j} n_{k}+\delta_{j k} n_{i} n_{l}+\delta_{j l} n_{i} n_{k}\right) \\
-15\left(3-7 \rho^{2}\right) n_{i} n_{j} n_{k} n_{l} & \text { for } x>a \\
-\frac{8 \pi}{15}\left(\delta_{i j} \delta_{k l}+\delta_{i k} \delta_{j l}+\delta_{i l} \delta_{j k}\right), & \text { for } x \leq a
\end{array}\right. \\
& \Psi_{p, i j}= \begin{cases}\frac{4 \pi}{105} a^{3} \rho^{2}\left[\begin{array}{c}
\left(7-3 \rho^{2}\right)\left(\delta_{i j} n_{p}+\delta_{i p} n_{j}+\delta_{j p} n_{i}\right) \\
-3\left(7-5 \rho^{2}\right) n_{i} n_{j} n_{p}
\end{array}\right], & \text { for } x>a \\
\frac{4 \pi}{105}\left[\left(7 a^{2}-3 x^{2}\right)\left(\delta_{i j} x_{p}+\delta_{i p} x_{j}+\delta_{j p} x_{i}\right)-6 x_{i} x_{j} x_{p}\right], & \text { for } x \leq a\end{cases} \\
& o \Psi_{p, i j k}=\left\{\begin{array}{cc}
\left(7-3 \rho^{2}\right)\left(\delta_{i j} \delta_{k p}+\delta_{i k} \delta_{j p}+\delta_{j k} \delta_{i p}\right) \\
\frac{4 \pi}{105} \rho^{3} a^{2}\left[\begin{array}{c} 
\\
-3\left(7-5 \rho^{2}\right)\left(\delta_{i j} n_{k} n_{p}+\delta_{k p} n_{i} n_{j}+\delta_{i k} n_{j} n_{p}\right) \\
-3\left(7-5 \rho^{2}\right)\left(\delta_{i p} n_{j} n_{k}+\delta_{j k} n_{i} n_{p}+\delta_{j p} n_{i} n_{k}\right) \\
+105\left(1-\rho^{2}\right) n_{i} n_{j} n_{k} n_{p}
\end{array}\right], \quad \text { for } x>a \\
\frac{4 \pi}{105}\left[\begin{array}{c}
\left(7 a^{2}-3 x^{2}\right)\left(\delta_{i j} \delta_{k p}+\delta_{i k} \delta_{j p}+\delta_{j k} \delta_{i p}\right) \\
-6\left(\delta_{i j} x_{k} x_{p}+\delta_{k p} x_{i} x_{j}+\delta_{i k} x_{j} x_{p}\right) \\
-6\left(\delta_{i p} x_{j} x_{k}+\delta_{j k} x_{i} x_{p}+\delta_{j p} x_{i} x_{k}\right)
\end{array}\right], & \text { for } x \leq a
\end{array}\right. \\
& \Psi_{p, i j k l}=\left\{\begin{array}{c}
-\left(7-5 \rho^{2}\right)\left(\delta_{i j} \delta_{k l}+\delta_{i k} \delta_{j l}+\delta_{i l} \delta_{j k}\right) n_{p} \\
-\left(7-5 \rho^{2}\right)\left(\delta_{j p} \delta_{k l}+\delta_{k p} \delta_{j l}+\delta_{l p} \delta_{j k}\right) n_{i} \\
-\left(7-5 \rho^{2}\right)\left(\delta_{i p} \delta_{k l}+\delta_{i k} \delta_{l p}+\delta_{i l} \delta_{k p}\right) n_{j} \\
-\left(7-5 \rho^{2}\right)\left(\delta_{i j} \delta_{l p}+\delta_{i p} \delta_{j l}+\delta_{i l} \delta_{j p}\right) n_{k} \\
-\left(7-5 \rho^{2}\right)\left(\delta_{i j} \delta_{k p}+\delta_{i k} \delta_{j p}+\delta_{i p} \delta_{j k}\right) n_{l} \\
+35\left(1-\rho^{2}\right)\left(\delta_{i j} n_{k} n_{l} n_{p}+\delta_{i k} n_{j} n_{l} n_{p}+\delta_{i l} n_{k} n_{k} n_{p}\right) \\
+35\left(1-\rho^{2}\right)\left(\delta_{i p} n_{j} n_{k} n_{l}+\delta_{j k} n_{i} n_{l} n_{p}+\delta_{j l} n_{i} n_{k} n_{p}\right) \\
+35\left(1-\rho^{2}\right)\left(\delta_{j p} n_{i} n_{k} n_{l}+\delta_{k l} n_{i} n_{j} n_{p}+\delta_{k p} n_{i} n_{j} n_{l}\right) \\
+35\left(1-\rho^{2}\right)\left(\delta_{l p} n_{i} n_{j} n_{k}\right)-35\left(7-9 \rho^{2}\right) n_{i} n_{j} n_{k} n_{l} n_{p}
\end{array}\right], \quad \text { for } r>a
\end{aligned}
$$


where

$$
x=\left(x_{i} x_{i}\right)^{\frac{1}{2}}, \quad \rho=\frac{a}{x}, \quad n_{i}=\frac{x_{i}}{x}
$$

\section{References}

[1] Perevozchikov, A., Yakovlev, G. and Kodolov, V.. Polyethylene foam waste utilization for light-weight concrete production. International Journal of Polymeric Materials 47.1 (2000): 7-17.

[2] Laukaitis, A., Zurauskas, R. and Kerien, J.. The effect of foam polystyrene granules on cement composite properties. Cement and Concrete Composites 27.1 (2005): 41-47.

[3] Mounanga, P., Gbongbon, W., Poullain, P. and Turcry, P.. Proportioning and characterization of lightweight concrete mixtures made with rigid polyurethane foam wastes. Cement and Concrete Composites 30.9 (2008): 806-814.

[4] Eaves, D.. Handbook of Polymer Foams. Polimeri 25 (2004): 1-2.

[5] Yin, H. M., Yang, D. J., Kelly, G. and Garant, J.. Design and performance of a novel building integrated PV/thermal system for energy efficiency of buildings. Solar Energy 87 (2013): 184195.

[6] Yang, D. J., Yuan, Z. F., Lee, P. H. and Yin, H. M.. Simulation and experimental validation of heat transfer in a novel hybrid solar panel. International Journal of Heat and Mass Transfer 55.4 (2012): 1076-1082.

[7] Yang, D. J., and Yin, H. M.. Energy conversion efficiency of a novel hybrid solar system for photovoltaic, thermoelectric, and heat utilization. Energy Conversion, IEEE Transactions on 26.2 (2011): 662-670.

[8] Christensen, R. M., and Lo, K. H.. Solutions for effective shear properties in three phase sphere and cylinder models. Journal of the Mechanics and Physics of Solids 27.4 (1979): 315-330.

[9] Gusev, Andrei A. Representative volume element size for elastic composites: a numerical study. Journal of the Mechanics and Physics of Solids 45.9 (1997): 1449-1459.

[10] Kanit, T., Forest, S., Galliet, I., Mounoury, V. and Jeulin, D.. Determination of the size of the representative volume element for random composites: statistical and numerical approach. International Journal of solids and structures 40.13 (2003): 3647-3679.

[11] Aboudi, J., Arnold, S. M. and Bednarcyk, B. A.. Micromechanics of Composite Materials: a Generalized Multiscale Analysis Approach. Butterworth-Heinemann (2012). 
[12] Fish, J.. Discrete-to-continuum scale bridging. Multiscaling in Molecular and Continuum Mechanics: Interaction of Time and Size from Macro to Nano. Springer Netherlands (2007): 85-102.

[13] Ma, H., Guo, Z., and Qin, Q. H.. Two-dimensional polynomial eigenstrain formulation of boundary integral equation with numerical verification. Applied Mathematics and Mechanics 32 (2011): 551-562.

[14] Ma, H., Yan, C., and Qin, Q. H.. Eigenstrain formulation of boundary integral equations for modeling particle-reinforced composites. Engineering analysis with boundary elements 33.3 (2009): 410-419.

[15] ACI Committee 213. Guide for Structural Lightweight Aggregate Concrete. ACI Journal Proceedings 64.8 (1967).

[16] Bonakdar, A., Babbitt, F., and Mobasher, B.. Physical and mechanical characterization of fiber-reinforced aerated concrete (FRAC). Cement and Concrete Composites 38 (2013): 8291

[17] Kockal, N. U. and Ozturan, T.. Strength and elastic properties of structural lightweight concretes. Materials and Design 32.4 (2011): 2396-2403.

[18] Zhang, M. H. and Gjvorv, O. E.. Mechanical properties of high-strength lightweight concrete. ACI Materials Journal 88, 3 (1991).

[19] Yasar, E., Atis, C. D., Kilic, A. and Gulsen, H.. Strength properties of lightweight concrete made with basaltic pumice and fly ash . Materials Letters 57.15 (2003): 2267-2270.

[20] Hill, R.. Elastic properties of reinforced solids: some theoretical principles. Journal of the Mechanics and Physics of Solids 11.5 (1963): 357-372.

[21] Willis, J.R.. Variational principles for dynamic problems for inhomogeneous elastic media . Wave Motion 3.1 (1981): 1-11.

[22] Mura, T.. Micromechanics of Defects in Solids. Kluwer Academic Publishers, Dordrecht (1987).

[23] Nemat-Nasser, S. and Hori, M.. Micromechanics: Overall Properties of Heterogeneous Materials. Elsevier (1999).

[24] Yin, H. M., Sun, L. Z. and Chen, J. S.. Micromechanics-based hyperelastic constitutive modeling of magnetostrictive particle-filled elastomers. Mechanics of materials 34.8 (2002): 505516. 
[25] Xia, Z.H., Zhang, Y. F. and Ellyin, F.. A unified periodical boundary conditions for representative volume elements of composites and applications. International Journal of Solids and Structures 40.8 (2003): 1907-1921.

[26] Yin, H. M. and Sun, L. Z.. Elastic modelling of periodic composites with particle interactions. Philosophical magazine letters 85.4 (2005): 163-173.

[27] Reiter, T., Dvorak, G. J. and Tvergaard, V.. Micromechanical models for graded composite materials . Journal of the Mechanics and Physics of Solids 45.8 (1997): 1281-1302.

[28] Yin, H. M., Sun, L. Z. and Paulino, G. H.. Micromechanics-based elastic model for functionally graded materials with particle interactions. Acta Materialia 52.12 (2004): 3535-3543.

[29] Yin, H. M., Paulino, G. H., Buttlar, W. G. and Sun, L. Z.. Micromechanics-based thermoelastic model for functionally graded particulate materials with particle interactions. Journal of the Mechanics and Physics of Solids 55.1 (2007): 132-160.

[30] Eshelby, J. D.. The determination of the elastic field of an ellipsoidal inclusion, and related problems. Proceedings of the Royal Society of London. Series A. Mathematical and Physical Sciences 241.1226 (1957): 376-396.

[31] Eshelby, J. D.. The elastic field outside an ellipsoidal inclusion. Proceedings of the Royal Society of London. Series A. Mathematical and Physical Sciences 252.1271 (1959): 561569.

[32] Voigt, W.. Ueber die Beziehung zwischen den beiden Elasticitätsconstanten isotroper Körper. Annalen der Physik 274.12 (1889): 573-587.

[33] Reuss, A. Berechnung der Fliegrenze von Mischkristallen auf Grund der Plastizittsbedingung fr Einkristalle. ZAMM-Journal of Applied Mathematics and Mechanics/Zeitschrift fr Angewandte Mathematik und Mechanik 9.1 (1929): 49-58.

[34] Budiansky, B.. On the elastic moduli of some heterogeneous materials. Journal of the Mechanics and Physics of Solids 13.4 (1965): 223-227.

[35] Mori, T and Tanaka, K.. Average stress in matrix and average elastic energy of materials with misfitting inclusions. Acta metallurgica 21.5 (1973): 571-574.

[36] Hill, R.. A self-consistent mechanics of composite materials. Journal of the Mechanics and Physics of Solids 13.4 (1965): 213-222.

[37] Norma, A. S. T. M.. C39M. Standard Test Method for Compressive Strength of Cylindrical Concrete Specimens. ASTM International (2005). 
[38] Kröner, E.. Modified Green functions in the theory of heterogeneous and/or anisotropic linearly elastic media. Micromechanics and inhomogeneity, Springer New York (1990): 197211.

[39] Yin, H. M., Lee, P. H. and Liu, Y. J.. Equivalent inclusion method for the Stokes flow of drops moving in a viscous fluid. Journal of Applied Mechanics 81.7 (2014): 071010.

[40] Liu, Y. J. and Yin, H. M.. Equivalent inclusion method-based simulation of particle sedimentation toward functionally-graded material manufacturing. Acta Mechanica 225.4-5 (2014): 1429-1445.

[41] Yin, H. M., and Sun, L. Z.. Magnetoelastic modelling of composites containing randomly dispersed ferromagnetic particles. Philosophical Magazine 86.28 (2006): 4367-4395.

[42] Yin, H. M., Sun, L. Z. and Chen, J. S.. Magneto-elastic modeling of composites containing chain-structured magnetostrictive particles. Journal of the Mechanics and Physics of Solids 54.5 (2006): 975-1003.

[43] Cormen, T. H., Leiserson, C. E., Rivest, R. L. and Stein, C.. Introduction to algorithms. Vol. 2. Cambridge: MIT press, 2001. 